\title{
Phase coexistence in the hard-sphere Yukawa chain fluid with chain length polydispersity: High temperature approximation
}

\author{
S. P. Hlushak, Yu. V. Kalyuzhnyi \\ Institute for Condensed Matter Physics, Svientsitskoho 1, 79011 Lviv, Ukraine
}

(Dated: July 30, 2018)

\begin{abstract}
High temperature approximation (HTA) is used to describe the phase behavior of polydisperse multi-Yukawa hard-sphere chain fluid mixtures with chain length polydispersity. It is demonstrated that in the frames of the HTA the model belongs to the class of "truncatable free energy models", i.e. the models with thermodynamical properties (Helmholtz free energy, chemical potential and pressure) defined by the finite number of generalized moments. Using this property we were able to calculate the complete phase diagram (i.e., cloud and shadow curves as well as binodals) and chain length distribution functions of the coexisting phases.
\end{abstract}

\section{INTRODUCTION}

In this paper we continue our study of the phase behavior of polydisperse fluid mixtures. In the previous studies [1, 2, 3, 4, 5] a scheme based on integral equation theory that allows one to include correlation between the particles on the level of the mean spherical approximation (MSA) has been developed. Usually the theoretical concepts used to study polydisperse systems treat such systems as a mixture with an infinite number of components, each of them characterized by a (continuous) variable $x$, which is distributed according to the distribution function $f(x)$. In generalizing the formalism of the MSA for a mixture with a finite number of components to the polydisperse case, we obtained expressions for the structural and thermodynamic properties that can be expressed via a finite number of generalized moments of the distribution function $f(x)$. Such models are called truncatable free energy (TFE) models and have the attractive feature that the formally infinitely many coexistance equations for phase equilibrium can be mapped onto a finite number of coupled, highly non-linear equations in these moments. Polydisperse mixture of hardsphere Yukawa [1, 5] and charged hard-sphere fluid [2, 3, 4] have been studied via this approach. In the case of Yukawa mixtures application of the MSA is restricted to the systems with factorized version of the one-Yukawa interaction, i.e. the matrix of the coefficients describing the strength of the corresponding interaction is factorized into the product of two vectors. To remove this restriction recently high temperature approximation (HTA) for polydisperse multi-Yukawa hard-sphere fluid has been developed [6]. It was demonstrated that within HTA polydisperse multi-Yukawa hardsphere model belongs to the family of truncatable free energy models. Unlike MSA, HTA includes correlations at the level of the reference system and in general it is less accurate than MSA, especially at lower densities [7, 8]. However, HTA can be used to describe the phase behavior of a polydisperse hard-sphere Yukawa mixture with arbitrary number of non-factorized Yukawa tails. This feature makes the number of the systems amenable to HTA description much larger than the number of the systems amenable to MSA description.

In this paper we are focused on the phase behavior of polydisperse mixture of Yukawa hard-sphere chain molecules with polydispersity in the chain length. Our study is based on the extension of the recently proposed HTA for the model at hand. Laplace transforms of the site-site radial distribution functions (RDF) of the reference system, which are needed as an input, are calculated using product reactant Ornstein-Zernike approach (PROZA) 9, 10, 11], appropriately reformulated in terms of the average total and direct correlation functions. For thermodynamical properties of the reference system we have used thermodynamic perturbation theory of Wertheim [12, 13], generalized for polydisperse hard-sphere chain mixture. Similar as in the case of polydisperse hard-sphere Yukawa mixture polydisperse hard-sphere Yukawa chain mixture within present version of the HTA belongs to the family of TFE models. This feature allows us to use a scheme developed earlier [1, 2, 3, 4] and to calculate complete phase diagram of the model (i.e., cloud and shadow curves as well as binodals) and chain-length distribution functions of the coexisting phases.

\section{HARD-SPHERE YUKAWA CHAIN MODEL}

We consider mixture of freely jointed tangent hard-sphere Yukawa chain molecules of $N$ different chain species with densities $\rho_{a}$ and chain lengths $m_{a}$. Here and below small Latin letters $a, b, c$ will denote species of chain molecules that take values $1 . . N$, and small letters $i, j, k, \ldots$ will indicate monomer species of the chain, which take values $1, \ldots, m_{a}$. Monomers in each chain are assumed to be of the same size $R^{a}$. In addition to the hard-sphere interaction Yukawa potential is acting between monomers, which are not connected directly. The resulting hard-sphere Yukawa potential 
reads

$$
u_{Y}^{a b}(r)= \begin{cases}\infty, & 0 \leq r \leq R^{a b} \\ -\frac{\epsilon_{0} r_{0}}{r} e^{-z^{(0)}\left(r-R^{a b}\right)}, & R^{a b}<r\end{cases}
$$

where $\epsilon_{0}$ represents energy at contact, $z^{(0)}$ is Yukawa screening length and $r_{0}$ is distance unit. For simplicity reasons we have employed only one-Yukawa interaction, but our theory can be easily extended by multi-Yukawa potential, which can mimic large variety of realistic potentials.

\section{STRUCTURE PROPERTIES OF THE REFERENCE SYSTEM}

For the sake of simplicity instead of the regular site-site $\mathrm{RDF} g_{i j}^{a b}(r)$ we are using here average site-site $\mathrm{RDF} g^{a b}(r)$, defined as follows:

$$
\bar{g}^{a b}(r)=\frac{1}{m_{a} m_{b}} \sum_{i j} g_{i j}^{a b}(r)
$$

These RDFs are calculated from the averaged version of the multidensity OZ equation, derived below.

\section{A. Averaged version of the multidensity $\mathrm{OZ}$ equation in the ideal chain approximation.}

Multidensity OZ equation for the multicomponent chain fluid in the ideal chain approximation is [9, 10, 11]:

$$
\mathbf{h}_{i j}^{a b}(r)=\mathbf{c}_{i j}^{a b}(r)+\sum_{c, k} \int\left[\mathbf{c}_{i k}^{a c}\left(r^{\prime}\right)+\boldsymbol{\Delta}_{i k}^{a c}\left(r^{\prime}\right)\right] \rho_{c} \alpha\left[\mathbf{h}_{k j}^{c b}\left(\left|\mathbf{r}-\mathbf{r}^{\prime}\right|\right)+\boldsymbol{\Delta}_{k j}^{c b}\left(\left|\mathbf{r}-\mathbf{r}^{\prime}\right|\right)\right] d \mathbf{r}^{\prime}
$$

where we have extracted from the correlation functions the terms with delta functions and denoted them by $\boldsymbol{\Delta}_{i j}^{a b}(r)$. The matrices used in (3) are total correlation function matrix

$$
\mathbf{h}_{i j}^{a b}(r)=\left(\begin{array}{ccc}
h_{i j, 00}^{a b}(r) & h_{i j, 0 A}^{a b}(r) & h_{i j, 0 B}^{a b}(r) \\
h_{i j, A 0}^{a b}(r) & h_{i j, A A}^{a b}(r) & h_{i j, A B}^{a b}(r) \\
h_{i j, B 0}^{a b}(r) & h_{i j, B A}^{a b}(r) & h_{i j, B B}^{a b}(r)
\end{array}\right)
$$

direct correlation function matrix

$$
\mathbf{c}_{i j}^{a b}(r)=\left(\begin{array}{ccc}
c_{i j, 00}^{a b}(r) & c_{i j, 0 A}^{a b}(r) & c_{i j, 0 B}^{a b}(r) \\
c_{i j, A 0}^{a b}(r) & c_{i j, A A}^{a b}(r) & c_{i j, A B}^{a b}(r) \\
c_{i j, B 0}^{a b}(r) & c_{i j, B A}^{a b}(r) & c_{i j, B B}^{a b}(r)
\end{array}\right)
$$

density matrix

$$
\alpha=\left(\begin{array}{lll}
1 & 1 & 1 \\
1 & 0 & 1 \\
1 & 1 & 0
\end{array}\right)
$$

and the matrix containing delta-functions:

$$
\boldsymbol{\Delta}_{i j}^{a b}(r)=\delta_{i, j+1}\left(\begin{array}{ccc}
0 & 0 & 0 \\
0 & 0 & \Delta^{a b}(r) \\
0 & 0 & 0
\end{array}\right)+\delta_{i, j-1}\left(\begin{array}{ccc}
0 & 0 & 0 \\
0 & 0 & 0 \\
0 & \Delta^{a b}(r) & 0
\end{array}\right)
$$

where

$$
\Delta^{a b}(r)=\frac{\delta_{a b}}{4 \pi \rho_{a}\left(R^{a b}\right)^{2}} \delta\left(r-R^{a b}\right), \quad R^{a b}=\left(R^{a}+R^{b}\right) / 2
$$


Taking the average of both sides of equation (3) we have

$\frac{1}{m_{a} m_{b}} \sum_{i j} \mathbf{h}_{i j}^{a b}(r)=\frac{1}{m_{a} m_{b}} \sum_{i j} \mathbf{c}_{i j}^{a b}(r)+\frac{1}{m_{a} m_{b}} \sum_{i j} \sum_{c k} \int\left[\mathbf{c}_{i k}^{a c}\left(r^{\prime}\right)+\boldsymbol{\Delta}_{i k}^{a c}\left(r^{\prime}\right)\right] \rho_{c} \alpha\left[\mathbf{h}_{k j}^{c b}\left(\left|\mathbf{r}-\mathbf{r}^{\prime}\right|\right)+\boldsymbol{\Delta}_{k j}^{c b}\left(\left|\mathbf{r}-\mathbf{r}^{\prime}\right|\right)\right] d \mathbf{r}^{\prime}$.

By direct multiplication and summation, it can be shown, that

$$
\sum_{i j} \sum_{c k} \boldsymbol{\Delta}_{i k}^{a c}\left(r^{\prime}\right) \rho_{c} \alpha \boldsymbol{\Delta}_{k j}^{c b}\left(\left|\mathbf{r}-\mathbf{r}^{\prime}\right|\right)=\left(m_{a}-2\right) \sum_{c} \bar{\Delta}^{a c}\left(r^{\prime}\right) \rho_{c} \alpha \overline{\boldsymbol{\Delta}}^{c b}\left(\left|\mathbf{r}-\mathbf{r}^{\prime}\right|\right)
$$

where we have introduced new matrix

$$
\overline{\boldsymbol{\Delta}}^{a b}(r)=\left(\begin{array}{ccc}
0 & 0 & 0 \\
0 & 0 & \Delta^{a b}(r) \\
0 & \Delta^{a b}(r) & 0
\end{array}\right)
$$

Similarly

$$
\begin{aligned}
& \sum_{i j} \sum_{c k} \boldsymbol{\Delta}_{i k}^{a c}\left(r^{\prime}\right) \rho_{c} \alpha \mathbf{h}_{k j}^{c b}\left(\left|\mathbf{r}-\mathbf{r}^{\prime}\right|\right)=\sum_{c}\left(m_{c}-1\right) m_{b} \overline{\boldsymbol{\Delta}}^{a c}\left(r^{\prime}\right) \rho_{c} \alpha \overline{\mathbf{h}}^{c b}\left(\left|\mathbf{r}-\mathbf{r}^{\prime}\right|\right), \\
& \sum_{i j} \sum_{c k} \mathbf{c}_{i k}^{a c}\left(r^{\prime}\right) \rho_{c} \alpha \boldsymbol{\Delta}_{k j}^{c b}\left(\left|\mathbf{r}-\mathbf{r}^{\prime}\right|\right)=\sum_{c} m_{a}\left(m_{c}-1\right) \overline{\mathbf{c}}^{a c}\left(r^{\prime}\right) \rho_{c} \alpha \overline{\boldsymbol{\Delta}}^{c b}\left(\left|\mathbf{r}-\mathbf{r}^{\prime}\right|\right),
\end{aligned}
$$

where $\overline{\mathbf{c}}^{c b}(r)$ and $\overline{\mathbf{h}}^{c b}(r)$ are averaged direct and total correlation functions

$$
\begin{aligned}
\overline{\mathbf{c}}^{a b}(r) & =\frac{1}{m_{a} m_{b}} \sum_{i j} \mathbf{c}_{i k}^{a b}(r), \\
\overline{\mathbf{h}}^{a b}(r) & =\frac{1}{m_{a} m_{b}} \sum_{i j} \mathbf{h}_{i k}^{a b}(r) .
\end{aligned}
$$

It should be noted that $\overline{\boldsymbol{\Delta}}^{a b}(r)$ has a Kroneker delta symbol $\delta_{a b}$, thus sums over $c$ in (12) and (13) can be removed. They are kept, because they will be used to assemble the OZ equation from the corresponding averaged quantities.

$$
\begin{aligned}
\overline{\mathbf{h}}^{a b}(r)= & \overline{\mathbf{c}}^{a b}(r)+\sum_{c} \rho_{c} m_{c} \int\left[\overline{\mathbf{c}}^{a c}\left(r^{\prime}\right) \alpha \overline{\mathbf{h}}^{c b}\left(\left|\mathbf{r}-\mathbf{r}^{\prime}\right|\right)+\frac{m_{c}-1}{m_{c}} \overline{\mathbf{c}}^{a c}\left(r^{\prime}\right) \alpha \bar{\Delta} c b\left(\left|\mathbf{r}-\mathbf{r}^{\prime}\right|\right)+\right. \\
& \left.\frac{m_{c}-1}{m_{c}} \bar{\Delta}_{i k}^{a c}\left(r^{\prime}\right) \alpha \overline{\mathbf{h}}_{k j}^{c b}\left(\left|\mathbf{r}-\mathbf{r}^{\prime}\right|\right)+\frac{m_{c}-2}{m_{c}} \bar{\Delta}_{i k}^{a c}\left(r^{\prime}\right) \alpha \bar{\Delta}_{k j}^{c b}\left(\left|\mathbf{r}-\mathbf{r}^{\prime}\right|\right)\right] d \mathbf{r}^{\prime}
\end{aligned}
$$

Neglecting the terms with $1 /\left(m_{c}\right)^{2}$ equation (16) can be factorized

$$
\overline{\mathbf{h}}^{a b}(r)=\overline{\mathbf{c}}^{a b}(r)+\sum_{c} \rho_{c} m_{c} \int\left[\overline{\mathbf{c}}^{a c}\left(r^{\prime}\right)+\frac{m_{c}-1}{m_{c}} \overline{\boldsymbol{\Delta}}^{a c}\left(r^{\prime}\right)\right] \alpha\left[\overline{\mathbf{h}}^{c b}\left(\left|\mathbf{r}-\mathbf{r}^{\prime}\right|\right)+\frac{m_{c}-1}{m_{c}} \bar{\Delta}^{c b}\left(\left|\mathbf{r}-\mathbf{r}^{\prime}\right|\right)\right] d \mathbf{r}^{\prime}
$$

and all the terms containing delta-functions can be included into corresponding correlation functions

$$
\mathbf{h}^{a b}(r)=\mathbf{c}^{a b}(r)+\sum_{c} \rho_{c} m_{c} \int \mathbf{c}^{a c}\left(r^{\prime}\right) \alpha \mathbf{h}^{c b}\left(\left|\mathbf{r}-\mathbf{r}^{\prime}\right|\right) d \mathbf{r}^{\prime}
$$

where 


$$
\begin{aligned}
\mathbf{c}^{a b}(r) & =\overline{\mathbf{c}}^{a c}(r)+\frac{m_{c}-1}{m_{c}} \bar{\Delta}^{a c}(r), \\
\mathbf{h}^{a b}(r) & =\overline{\mathbf{h}}^{c b}(r)+\frac{m_{c}-1}{m_{c}} \overline{\boldsymbol{\Delta}}^{c b}(r),
\end{aligned}
$$

are new averaged correlation functions which now depends only on chain species index, and are regarded to be the same for all beads of the chain. Also, new averaged contribution from sticky interaction (11) was obtained, and it will be used now in formulation of PPY closure condition in terms of partial correlation functions $h_{\alpha \beta}^{a b}(r)$ and $c_{\alpha \beta}^{a b}(r)$

$$
\begin{aligned}
h_{\alpha \beta}^{a b}(r) & =-\delta_{\alpha 0} \delta_{\beta 0}, \quad r<R^{a b}, \\
c_{\alpha \beta}^{a b}(r) & =\frac{\delta_{a b}\left(m_{a}-1\right)}{4 \pi \rho_{a} m_{a}\left(R^{a b}\right)^{2}}\left(\delta_{\alpha A} \delta_{\beta B}+\delta_{\alpha B} \delta_{\beta A}\right) \delta\left(r-R^{a b}\right), \quad r \geq R^{a b} .
\end{aligned}
$$

As one can see the above closure conditions contain factor $\frac{m_{c}-1}{m_{c}}$ which appears due to averaging of the $\mathrm{OZ}$ equation. It appears that the averaged version of the $\mathrm{OZ}$ equation (18) coincide with multidensity $\mathrm{OZ}$ equation for the fluid of associating particles forming a chains with the average length $m_{a}[15,16]$.

\section{B. Solution of the averaged $\mathrm{OZ}$ equation}

Solution of the set of the OZ equation (18) with closure conditions (21) and (22) was performed employing Baxter factorization method [17]. Factorizing this set of equations (18) we have

$$
\begin{aligned}
& -r c_{\alpha \beta}^{a b}(r)=\left[q_{\alpha \beta}^{a b}(r)\right]^{\prime}-2 \pi \sum_{c} \rho_{c} m_{c} \sum_{\gamma, \delta}\left(1-\delta_{\gamma \delta}+\delta_{\gamma 0} \delta_{\delta 0}\right) \frac{\partial}{\partial r} \int_{S^{a c}}^{\min \left[R^{a c}, R^{c b}-r\right]} \quad q_{\gamma \alpha}^{c a}(t) q_{\delta \beta}^{c b}(r+t) d t \\
& \quad f o r \quad S^{a b}<r<R^{a b}, \\
& -r h_{\alpha \beta}^{a b}(r)=\left[q_{\alpha \beta}^{a b}(r)\right]^{\prime}-2 \pi \sum_{c} \rho_{c} m_{c} \sum_{\gamma, \delta}\left(1-\delta_{\gamma \delta}+\delta_{\gamma 0} \delta_{\delta 0}\right) \int_{S^{a c}}^{R^{a c}} q_{\alpha \gamma}^{a c}(t)(r-t) h_{\delta \beta}^{c b}(|r-t|) d t, \quad r>S^{a b},
\end{aligned}
$$

where $S^{a b}=\frac{1}{2}\left(R^{(a)}-R^{(b)}\right)$, and $q$ is Baxter function

$$
q_{\alpha \beta}^{a b}(r)=\left(\frac{1}{2} a_{\alpha}^{(a)} r^{2}+b_{\alpha}^{(a)} r\right) \delta_{\beta 0}+d_{\alpha \beta}^{a b},
$$

defined in interval $S^{a b}<r<R^{a b}$, with coefficients $a_{\alpha}^{(a)}, b_{\alpha}^{(a)}$ and $d_{\alpha \beta}^{a b}$ found to be

$$
\begin{aligned}
a_{0}^{(a)} & =\frac{1}{\Delta}+\frac{1}{2} \frac{R^{(a)} \xi_{2}}{\Delta^{2}} \\
b_{0}^{(a)} & =-\frac{1}{4} \frac{\left(R^{(a)}\right)^{2} \xi_{2}}{\Delta^{2}} \\
d_{00}^{a b} & =-\frac{1}{2} \frac{\left(R^{(a)}\right)^{2} \xi_{2}}{\Delta}+\frac{1}{4} \frac{R^{(a)} R^{a b} S^{a b} \xi_{2}}{\Delta^{2}}, \\
a_{M}^{(a)} & =-\frac{1}{2} \frac{\left(m_{a}-1\right)}{m_{a} \Delta}\left(\delta_{M A}+\delta_{M B}\right) \\
b_{M}^{(a)} & =-\frac{1}{2} a_{M}^{(a)} R^{(a)} \\
d_{M 0}^{a b} & =\frac{1}{2} a_{M}^{(a)} R^{a b} S^{a b} \\
d_{0 L}^{a b} & =0 \\
d_{M L}^{a b} & =\frac{\delta_{a b}}{4 \pi \rho^{a} m_{a} R^{(a)}}\left(\frac{m_{a}-1}{m_{a}}\right)\left(\delta_{M A} \delta_{L B}+\delta_{L A} \delta_{M B}\right)
\end{aligned}
$$


where $\xi_{i}=\pi \sum_{c} \rho_{c} m_{c}\left(R^{(c)}\right)^{i}$ and $\Delta=1-\xi_{3} / 6$.

The corresponding expressions for contact values of radial distribution function $g_{\alpha \beta}^{a b}(r)=\delta_{\alpha 0} \delta_{\beta 0}+h_{\alpha \beta}^{a b}(r)$ are as follows:

$$
\begin{aligned}
g_{00}^{a b}\left(R^{a b}+\right) & =\frac{1}{\Delta}+\frac{1}{4} \frac{R^{(a)} R^{(b)} \xi_{2}}{\Delta^{2} R^{a b}} \\
g_{M 0}^{a b}\left(R^{a b}+\right) & =-\frac{1}{4} \frac{R^{(b)}}{\Delta R^{a b}}\left(\frac{m_{a}-1}{m_{a}}\right) \\
g_{0 M}^{a b}\left(R^{a b}+\right) & =-\frac{1}{4} \frac{R^{(a)}}{\Delta R^{a b}}\left(\frac{m_{b}-1}{m_{b}}\right) \\
g_{M L}^{a b}\left(R^{a b}+\right) & =\frac{\delta_{a b}}{8 \pi \rho_{a} m_{a}\left(R^{(a)}\right)^{2} R^{a b}}\left(\frac{m_{a}-1}{m_{a}}\right)^{2}\left(1-\delta_{M L}\right)
\end{aligned}
$$

Note that $g_{M L}^{a a}(r)$ contains also delta function $\delta\left(r-R^{(a)}\right)$ which describes neighbor hard spheres of the chain.

\section{Laplace transforms of the site-site radial distribution functions}

Integrating both sides of equation (24) with $\int_{S^{a b}}^{\infty} e^{-s r} \ldots d r$ we have

$$
\begin{aligned}
& \sum_{c} \sum_{\delta}\left(\delta_{c a} \delta_{\delta \alpha}-2 \pi \rho_{c} m_{c}\left[Q_{\alpha 0}^{a c}(s)+\left(1-\delta_{\delta A}\right) Q_{\alpha A}^{a c}(s)+\left(1-\delta_{\delta B}\right) Q_{\alpha B}^{a c}(s)\right]\right) G_{\delta \beta}^{c b}(s)= \\
& \delta_{\alpha 0} \delta_{\beta 0} \int_{S^{a b}}^{\infty} e^{-s r} d r-Q_{\alpha \beta}^{a b^{\prime}}(s)-2 \pi \sum_{c} \rho_{c} m_{c} \sum_{\gamma \delta}\left(1-\delta_{\gamma \delta}+\delta_{\gamma 0} \delta_{\delta 0}\right) \delta_{\delta 0} \delta_{\beta 0} \int_{d t S^{a c}}^{R^{a c}} q_{\alpha \gamma}^{a c}(t)\left(\frac{1}{s^{2}}-\frac{S^{a b}}{s}-\frac{t}{s}\right) e^{-s S^{a b}}
\end{aligned}
$$

where Laplace transforms and their derivatives are denoted by capital letters:

$$
\begin{aligned}
Q_{\alpha \beta}^{a b}(s)= & \int_{S^{a b}}^{\infty} e^{-s r} q_{\alpha \beta}^{a b}(r) d r=e^{-s S^{a b}}\left(\left[a_{\alpha}^{(a)}\left(\varphi_{2}\left(R^{(b)}\right)+\frac{R^{(b)}}{2} \varphi_{1}\left(R^{(b)}\right)\right)+\left(\frac{a_{\alpha}^{(a)} R^{(a)}}{2}+b_{\alpha}^{(a)}\right) \varphi_{1}\left(R^{(b)}\right)\right] \delta_{\beta 0}(3)\right. \\
& \left.+\left(1-\delta_{\alpha 0}\right)\left(1-\delta_{\beta 0}\right) d_{\alpha \beta}^{a b} \varphi_{0}\left(R^{(b)}\right)\right) \\
Q_{\alpha \beta}^{a b^{\prime}}(s)= & \int_{S^{a b}}^{\infty} e^{-s r} q_{\alpha \beta}^{a b^{\prime}}(r) d r=e^{-s S^{a b}}\left(\left[a_{\alpha}^{(a)}\left(\varphi_{1}\left(R^{(b)}\right)+\frac{R^{(b)}}{2} \varphi_{0}\left(R^{(b)}\right)\right)+\left(\frac{a_{\alpha}^{(a)} R^{(a)}}{2}+b_{\alpha}^{(a)}\right) \varphi_{0}\left(R^{(b)}\right)\right] \delta_{\beta 0}\left(\delta_{\alpha 0}\right)\left(1-\delta_{\beta 0}\right) d_{\alpha \beta}^{a b}\right) \\
& -\left(1-\delta_{\alpha}\right.
\end{aligned}
$$

where

$$
\begin{aligned}
& \varphi_{2}\left(R^{(b)}\right)=\frac{1-s R^{(b)}+s^{2} R^{(b)^{2}} / 2-e^{-s R^{(b)}}}{s^{3}}, \\
& \varphi_{1}\left(R^{(b)}\right)=\frac{1-s R^{(b)}-e^{-s R^{(b)}}}{s^{2}}, \\
& \varphi_{0}\left(R^{(b)}\right)=\frac{1-e^{-s R^{(b)}}}{s},
\end{aligned}
$$

and

$$
G_{\alpha \beta}^{a b}(s)=\int_{S^{a b}}^{\infty} e^{-s r} g_{\alpha \beta}^{a b}(r) d r .
$$

Similarly as in [18, 19, 20], solution of equation (38) reduces to inverting Jacobi like matrix. Note that here we have infinite number of vectors forming the matrix. To describe briefly our method of matrix inversion, let us start with equation (38) rewritten in following form: 


$$
\sum_{c} \sum_{\delta} M_{\alpha \delta}^{a c} G_{\delta \beta}^{c b}(s)=\hat{a}_{\alpha}^{(a)}\left[\frac{1}{s^{2}}+\frac{R^{(b)}}{2 s}\right] \delta_{\beta 0}+\hat{c}_{\alpha}^{(a)} \frac{1}{s} \delta_{\beta 0}+\sum_{t} \hat{e}_{\alpha}^{(a)} \frac{\delta_{t b} \delta_{\beta A}}{2 \pi \rho_{b} m_{b}} e^{-s R^{(b)} / 2}+\sum_{t} \hat{g}_{\alpha}^{(a)} \frac{\delta_{t b} \delta_{\beta B}}{2 \pi \rho_{b} m_{b}} e^{-s R^{(b)} / 2},
$$

where Jacobi like matrix $M_{\alpha \delta}^{a c}$ is given by

$$
M_{\alpha \delta}^{a c}=\delta_{a c} \delta_{\alpha \delta}-\hat{a}_{\alpha}^{(a)} \hat{b}_{\delta}^{(c)}-\hat{c}_{\alpha}^{(a)} \hat{d}_{\delta}^{(c)}-\sum_{t}\left(\hat{e}_{\alpha}^{(a)(t)} \hat{f}_{\delta}^{(c)(t)}+\hat{g}_{\alpha}^{(a)(t)} \hat{h}_{\delta}^{(c)(t)}\right)
$$

where

$$
\begin{aligned}
& \hat{a}_{\alpha}^{(a)}=e^{-s R^{(a)} / 2} a_{\alpha}^{(a)}, \\
& \hat{b}_{\delta}^{(c)}=2 \pi \rho_{c} m_{c}\left[\varphi_{2}\left(R^{(c)}\right)+\frac{R^{(c)}}{2} \varphi_{1}\left(R^{(c)}\right)\right] e^{s R^{(c)} / 2}, \\
& \hat{c}_{\alpha}^{(a)}=e^{-s R^{(a)} / 2}\left[\frac{R^{(a)}}{2} a_{\alpha}^{(a)}+b_{\alpha}^{(a)}\right]=e^{-s R^{(b)} / 2} \frac{\delta_{\alpha 0} R^{(a)}}{2 \Delta}, \\
& \hat{d}_{\delta}^{(c)}=2 \pi \rho_{c} m_{c} \varphi_{1}\left(R^{(c)}\right) e^{s R^{(c)} / 2}, \\
& \hat{e}_{\alpha}^{(a)(t)}=e^{-s R^{(a)} / 2} \frac{m_{a}-1}{2 m_{a} R^{(a)}} \delta_{a t} \delta_{\alpha B}, \\
& \hat{f}_{\delta}^{(c)(t)}=\varphi_{0}\left(R^{(c)}\right) e^{s R^{(c)} / 2} \delta_{c t}\left(1-\delta_{\delta A}\right), \\
& \hat{g}_{\alpha}^{(a)(t)}=e^{-s R^{(a)} / 2} \frac{m_{a}-1}{2 m_{a} R^{(a)}} \delta_{a t} \delta_{\alpha A}, \\
& \hat{h}_{\delta}^{(c)(t)}=\varphi_{0}\left(R^{(c)}\right) e^{s R^{(c)} / 2} \delta_{c t}\left(1-\delta_{\delta B}\right), \quad \text { for } \quad t=1 . . N .
\end{aligned}
$$

The last four vectors $\hat{e}_{\alpha}^{(a)(t)}, \hat{f}_{\delta}^{(c)(t)}, \hat{g}_{\alpha}^{(a)(t)}$ and $\hat{h}_{\delta}^{(c)(t)}$ appear due to decomposition of the Kronecker delta

$$
\delta_{a b}=\sum_{t} \delta_{a t} \delta_{t b}
$$

in $d_{\alpha \beta}^{a b}$. This decomposition makes matrix $M_{\alpha \delta}^{a c}$ Jacobi-like. In general it appears to be impossible to revert such matrix with arbitrary number of forming vectors $\hat{e}_{\alpha}^{(a)(t)}, \hat{f}_{\delta}^{(c)(t)}, \hat{g}_{\alpha}^{(a)(t)}$ and $\hat{h}_{\delta}^{(c)(t)}$ for $t=1 . . N$ without notion that some of these vectors are orthogonal and other can be composed back to form $\delta_{a b}$ :

$$
\begin{aligned}
\left(\hat{\mathbf{e}}^{(p)} \hat{\mathbf{f}}^{(q)}\right) & =\sum_{c} \sum_{\gamma} \hat{e}_{\gamma}^{(c)(p)} \hat{f}_{\gamma}^{(c)(q)}=\frac{m_{p}-1}{2 m_{p} R^{(p)}} \varphi_{0}\left(R^{(p)}\right) \delta_{p q}, \\
\left(\hat{\mathbf{e}}^{(p)} \hat{\mathbf{h}}^{(q)}\right) & =\sum_{c} \sum_{\gamma} \hat{e}_{\gamma}^{(c)(p)} \hat{h}_{\gamma}^{(c)(q)}=0, \\
\left(\hat{\mathbf{g}}^{(p)} \hat{\mathbf{f}}^{(q)}\right) & =\sum_{c} \sum_{\gamma} \hat{g}_{\gamma}^{(c)(p)} \hat{f}_{\gamma}^{(c)(q)}=0, \\
\left(\hat{\mathbf{g}}^{(p)} \hat{\mathbf{h}}^{(q)}\right) & =\sum_{c} \sum_{\gamma} \hat{g}_{\gamma}^{(c)(p)} \hat{h}_{\gamma}^{(c)(q)}=\frac{m_{p}-1}{2 m_{p} R^{(p)}} \varphi_{0}\left(R^{(p)}\right) \delta_{p q} .
\end{aligned}
$$

The inverse matrix was searched in the following form:

$$
\begin{aligned}
M_{\gamma \lambda}^{x y-1} & =\delta_{x y} \delta_{\gamma \lambda}+\alpha_{a b} \hat{a}_{\gamma}^{(x)} \hat{b}_{\lambda}^{(y)}+\alpha_{c d} \hat{c}_{\gamma}^{(x)} \hat{d}_{\lambda}^{(y)}+\alpha_{c b} \hat{c}_{\gamma}^{(x)} \hat{b}_{\lambda}^{(y)}+\alpha_{a d} \hat{a}_{\gamma}^{(x)} \hat{d}_{\lambda}^{(y)}+\sum_{t} \hat{a}_{\gamma}^{(x)}\left(\alpha_{a f}^{(t)} \hat{f}_{\lambda}^{(y)(t)}+\alpha_{a h}^{(t)} \hat{h}_{\lambda}^{(y)(t)}\right) \\
& +\sum_{t} \hat{c}_{\gamma}^{(x)}\left(\alpha_{c f}^{(t)} \hat{f}_{\lambda}^{(y)(t)}+\alpha_{c h}^{(t)} \hat{h}_{\lambda}^{(y)(t)}\right)+\sum_{t}\left(\alpha_{e b}^{(t)} \hat{e}_{\gamma}^{(x)(t)}+\alpha_{g b}^{(t)} \hat{g}_{\gamma}^{(x)(t)}\right) \hat{b}_{\lambda}^{(y)}+\sum_{t}\left(\alpha_{e d}^{(t)} \hat{e}_{\gamma}^{(x)(t)}+\alpha_{g d}^{(t)} \hat{g}_{\gamma}^{(x)(t)}\right) \hat{d}_{\lambda}^{(y)} \\
& +\sum_{t_{1}} \sum_{t_{2}} \alpha_{e f}^{\left(t_{1}\right)\left(t_{2}\right)} \hat{e}_{\gamma}^{(x)\left(t_{1}\right)} \hat{f}_{\lambda}^{(y)\left(t_{2}\right)}+\sum_{t_{1}} \sum_{t_{2}} \alpha_{g h}^{\left(t_{1}\right)\left(t_{2}\right)} \hat{g}_{\gamma}^{(x)\left(t_{1}\right)} \hat{h}_{\lambda}^{(y)\left(t_{2}\right)}+\sum_{t_{1}} \sum_{t_{2}} \alpha_{e h}^{\left(t_{1}\right)\left(t_{2}\right)} \hat{e}_{\gamma}^{(x)\left(t_{1}\right)} \hat{h}_{\lambda}^{(y)\left(t_{2}\right)} \\
& +\sum_{t_{1}} \sum_{t_{2}} \alpha_{g f}^{\left(t_{1}\right)\left(t_{2}\right)} \hat{g}_{\gamma}^{(x)\left(t_{1}\right)} \hat{f}_{\lambda}^{(y)\left(t_{2}\right)}
\end{aligned}
$$


where $\alpha_{a b}, \alpha_{c d}, \ldots, \alpha_{g f}^{\left(t_{1}\right)\left(t_{2}\right)}$ are unknown coefficients that follow from the relation $\mathbf{M M}^{-\mathbf{1}}=\mathbf{I}$ with $\mathbf{I}$ being unity matrix. We will skip these cumbersome calculations.

After receiving unknown coefficients $\alpha$ the inverse matrix was multiplied by the right hand side of equation (45) giving the following expressions for the Laplace transforms of the RDFs:

$$
\begin{aligned}
& G_{\alpha \beta}^{a b}(s)=\frac{e^{-s R^{a b}}}{\Delta D} \delta_{\alpha 0} \delta_{\beta 0}\left[\frac{1}{s^{2}}+\frac{R^{a b}}{s}-R^{a} R^{b} \sum_{c} \frac{\pi \rho_{c} m_{c}}{\Delta s^{2}} \frac{K^{(c)}}{1-K^{(c)}} R^{(c)} \frac{\varphi_{1}\left(R^{(c)}\right)}{\varphi_{0}\left(R^{(c)}\right)} s+\frac{R^{(a)} R^{(b)} \xi_{2}}{4 \Delta s}\right. \\
& \left.+\frac{1}{2 \Delta s^{2}} \sum_{c} \pi \rho_{c} m_{c} R^{(c)^{3}} \psi\left(R^{(c)}\right)+\sum_{c} \frac{\pi \rho_{c} m_{c} \varphi_{1}\left(R^{(c)}\right) \psi\left(R^{(c)}\right)}{2 \Delta s}\left(R^{(a)}-R^{(c)}\right)\left(R^{(b)}-R^{(c)}\right)\right] \\
& +\frac{e^{-s R^{a b}}}{\Delta D} \frac{K^{(a)}}{1-K^{(a)}}\left(1-\delta_{\alpha 0}\right) \delta_{\beta 0}\left[\frac{1}{s^{2}}+\frac{R^{a b}}{s}-\frac{R^{(a)}}{\varphi_{0}\left(R^{(a)}\right)}\left(\frac{1}{s^{2}}+\frac{R^{(b)}}{2 s}\right)-\frac{R^{(a)}}{2 \Delta s^{2}}\left(\xi_{2}-\sum_{c} \pi \rho_{c} m_{c} R^{(c)^{2}} \Theta\left(R^{(c)}\right)\right)\right. \\
& +\frac{R^{(a)} R^{(b)} \xi_{2}}{4 \Delta s}+\frac{1}{2 \Delta s^{2}} \frac{\varphi_{1}\left(R^{(a)}\right)}{\varphi_{0}\left(R^{(a)}\right)} s \sum_{c} \pi \rho_{c} m_{c} R^{(c)^{3}} \psi\left(R^{(c)}\right) \\
& \left.+\sum_{c} \frac{\pi \rho_{c} m_{c} \varphi_{1}\left(R^{(c)}\right)}{2 \Delta s}\left(R^{(a)} \Theta\left(R^{(c)}\right)-\frac{\varphi_{1}\left(R^{(a)}\right)}{\varphi_{0}\left(R^{(a)}\right)} s \psi\left(R^{(c)}\right) R^{(c)}\right)\left(R^{(b)}-R^{(c)}\right)\right] \\
& +\frac{e^{-s R^{a b}}}{\Delta D} \frac{K^{(b)}}{1-K^{(b)}} \delta_{\alpha 0}\left(1-\delta_{\beta 0}\right)\left[\frac{1}{s^{2}}+\frac{R^{a b}}{s}-\frac{R^{(b)}}{\varphi_{0}\left(R^{(b)}\right)}\left(\frac{1}{s^{2}}+\frac{R^{(a)}}{2 s}\right)-\frac{R^{(b)}}{2 \Delta s^{2}}\left(\xi_{2}-\sum_{c} \pi \rho_{c} m_{c} R^{(c)^{2}} \Theta\left(R^{(c)}\right)\right)\right. \\
& +\frac{R^{(a)} R^{(b)} \xi_{2}}{4 \Delta s}+\frac{1}{2 \Delta s^{2}} \frac{\varphi_{1}\left(R^{(b)}\right)}{\varphi_{0}\left(R^{(b)}\right)} s \sum_{c} \pi \rho_{c} m_{c} R^{(c)^{3}} \psi\left(R^{(c)}\right) \\
& \left.+\sum_{c} \frac{\pi \rho_{c} m_{c} \varphi_{1}\left(R^{(c)}\right)}{2 \Delta s}\left(R^{(b)} \Theta\left(R^{(c)}\right)-\frac{\varphi_{1}\left(R^{(b)}\right)}{\varphi_{0}\left(R^{(b)}\right)} s \psi\left(R^{(c)}\right) R^{(c)}\right)\left(R^{(a)}-R^{(c)}\right)\right] \\
& +\frac{e^{-s R^{a b}}}{\Delta D} \frac{K^{(a)}}{1-K^{(a)}} \frac{K^{(b)}}{1-K^{(b)}}\left(1-\delta_{\alpha 0}\right)\left(1-\delta_{\beta 0}\right)\left[\frac{\varphi_{1}\left(R^{(a)}\right)}{\varphi_{0}\left(R^{(a)}\right)} \frac{\varphi_{1}\left(R^{(b)}\right)}{\varphi_{0}\left(R^{(b)}\right)}+\left(\frac{R^{(b)}}{2} \frac{\varphi_{1}\left(R^{(a)}\right)}{\varphi_{0}\left(R^{(a)}\right)}+\frac{R^{(a)}}{2} \frac{\varphi_{1}\left(R^{(b)}\right)}{\varphi_{0}\left(R^{(b)}\right)}\right)\right. \\
& +\frac{R^{(a)} R^{(b)} \xi_{2}}{4 \Delta s}+\frac{1}{2 \Delta} \frac{\varphi_{1}\left(R^{(a)}\right)}{\varphi_{0}\left(R^{(a)}\right)} \frac{\varphi_{1}\left(R^{(b)}\right)}{\varphi_{0}\left(R^{(b)}\right)} \sum_{c} \pi \rho_{c} m_{c} R^{(c)^{3}} \psi\left(R^{(c)}\right) \\
& +\frac{s}{2 \Delta} \frac{\varphi_{1}\left(R^{(a)}\right)}{\varphi_{0}\left(R^{(a)}\right)} \frac{\varphi_{1}\left(R^{(b)}\right)}{\varphi_{0}\left(R^{(b)}\right)} \sum_{c} \pi \rho_{c} m_{c} \varphi_{1}\left(R^{(c)}\right) R^{(c)^{2}} \psi\left(R^{(c)}\right)+\frac{R^{(a)} R^{(b)}}{2 \Delta s} \sum_{c} \pi \rho_{c} m_{c} \varphi_{1}\left(R^{(c)}\right) \Theta\left(R^{(c)}\right) \\
& \left.-\frac{1}{2 \Delta}\left(R^{(b)} \frac{\varphi_{1}\left(R^{(a)}\right)}{\varphi_{0}\left(R^{(a)}\right)}+R^{(a)} \frac{\varphi_{1}\left(R^{(b)}\right)}{\varphi_{0}\left(R^{(b)}\right)}\right) \sum_{c} \pi \rho_{c} m_{c} \varphi_{1}\left(R^{(c)}\right) R^{(c)} \psi\left(R^{(c)}\right)\right] \\
& +e^{-s R^{(a)}} \frac{\delta_{a b}}{2 \pi \rho_{a} m_{a}} \frac{K^{(a)}}{1-K^{(a)}} \frac{1}{\varphi_{0}\left(R^{(a)}\right)}\left(\delta_{\alpha A} \delta_{\beta B}+\delta_{\alpha B} \delta_{\beta A}\right),
\end{aligned}
$$

where

$$
\begin{aligned}
K^{(a)} & =\left(\hat{\mathbf{g}}^{(a)} \hat{\mathbf{h}}^{(a)}\right)=\left(\hat{\mathbf{e}}^{(a)} \hat{\mathbf{f}}^{(a)}\right)=\frac{m_{a}-1}{2 m_{a} R^{(a)}} \varphi_{0}\left(R^{(a)}\right), \\
\psi\left(R^{(a)}\right) & =1+\frac{2 K^{(a)}}{1-K^{(a)}}, \\
\Theta\left(R^{(a)}\right) & =1+\frac{2 K^{(a)}}{1-K^{(a)}} \frac{\varphi_{1}\left(R^{(a)}\right)}{\varphi_{0}\left(R^{(a)}\right)} s,
\end{aligned}
$$


and

$$
\begin{aligned}
D= & 1-\frac{2}{\Delta} \sum_{t} \pi \rho_{t} m_{t} \varphi_{2}\left(R^{(t)}\right) \Theta\left(R^{(t)}\right)\left\{1+\frac{1}{2 \Delta} \sum_{c} \pi \rho_{c} m_{c} R^{(c)^{3}} \psi\left(R^{(c)}\right)\right\} \\
& \left.-\frac{1}{\Delta^{2}} \sum_{t} \pi \rho_{t} m_{t} \varphi_{2}\left(R^{(t)}\right) R^{(t)} \psi\left(R^{(t)}\right)\left\{\xi_{2}-\sum_{c} \pi \rho_{c} m_{c} R^{(c)}\right)^{2} \Theta\left(R^{(c)}\right)\right\} \\
& -\frac{2}{\Delta} \sum_{c} \pi \rho_{c} m_{c} \varphi_{1}\left(R^{(c)}\right)\left\{R^{(c)}\left(\psi\left(R^{(c)}\right)\left[\frac{1}{2}+\frac{\xi_{2} R^{(c)}}{4 \Delta}\right]+\frac{\Theta\left(R^{(c)}\right)}{2}\right)\right. \\
& \left.+\frac{1}{4 \Delta} \psi\left(R^{(c)}\right) \sum_{c} \pi \rho_{c} m_{c} \varphi_{1}\left(R^{(t)}\right) \Theta\left(R^{(c)}\right)\left(R^{(c)}-R^{(t)}\right)^{2}\right\} .
\end{aligned}
$$

Presented above Laplace transform of partial RDF is reperesented as a sum of five terms that are multiplied by delta symbols and thus some of them vanish for a ceratin values of the monomer state indices $\alpha$ and $\beta$. Laplace image of overall radial distribution function can be calculated by

$$
G_{\text {overall }}^{a b}(s)=\sum_{\alpha \beta} G_{\alpha \beta}^{a b}(s) .
$$

The first four terms of (61) contribute to intermolecular RDF, while the last term describes intramolecular RDF and contains Laplace transform of the delta-function. The intermolecular and intramolecular parts of the overall RDFs are related to partial RDFs by

$$
\begin{aligned}
& G_{i n t e r}^{a b}(s)=G_{00}^{a b}(s)+2 G_{A 0}^{a b}(s)+2 G_{0 A}^{a b}(s)+4 G_{A A}^{a b}(s), \\
& G_{\text {intra }}^{a b}(s)=2\left(G_{A B}^{a b}(s)-G_{A A}^{a b}(s)\right) .
\end{aligned}
$$

\section{THERMODYNAMIC PROPERTIES OF THE HARD-SPHERE YUKAWA CHAIN MIXTURE}

As in our previous paper [6], we use the HTA to obtain analytical expression for the Helmholtz free energy. Similarly as in the SAFT 13, 21] we assume that free energy per unit volume $f=A / V$ can be written as following

$$
f=f_{i d}+f_{H S}^{e x}+f_{H T A}+f_{\text {chain }}
$$

where $f_{i d}$ is the ideal gas contribution

$$
\beta f_{i d}=\sum_{c} \rho_{c} m_{c}\left[\ln \left(\Lambda_{c}^{3} \rho_{c}\right)-1\right]
$$

with $\Lambda_{c}$ being thermal de Broglie wavelength, $f_{H S}^{e x}$ is hard sphere excess free energy and $f_{H T A}$ is key HTA term that accounts for attractive interaction between the chain monomers and contains Yukawa potential averaged with respect to the structure of the reference system. Finally the third term in (69) contains contribution from the chain formation

$$
\beta f_{\text {chain }}=\sum_{c} \rho_{c}\left(m_{c}-1\right)\left[-\ln \left(4 \pi \rho_{c} R^{(c)^{2}} Y\right)+1\right],
$$

where $Y$ is hard-sphere cavity correlation function.

The hard sphere excess free energy $f_{H S}^{e x}$ and cavity correlation function $Y$ are taken in Mansoori-Carnahan-StarlingLeland approximation 22], which for hard sphere system of equal sizes are given by

$$
\begin{aligned}
\beta f_{H S}^{e x} & =\frac{1}{2 \pi \Delta} l^{2} R^{3}\left(1+\frac{1}{3 \Delta}\right), \\
Y & =\frac{1}{\Delta}+\frac{l R^{3}}{4 \Delta^{2}}+\frac{l^{2} R^{6}}{72 \Delta^{3}},
\end{aligned}
$$

where $R$ is the hard sphere diameter, $\Delta=1-l R^{3} / 6$ and $l=\pi \sum_{c} \rho^{c} m_{c}$ is the moment of the chain number densities. 
Key HTA term in (69) holds Yukawa attraction energy averaged with respect to the overall RDF of reference system

$$
\beta f_{H T A}=\beta \frac{2}{\pi} \sum_{a} \pi \rho_{a} m_{a} \sum_{b} \pi \rho_{b} m_{b} \int_{0}^{\infty} d r r^{2} u_{Y}^{a b}(r) g_{\text {overall }}^{a b}(r) .
$$

Due to the choice of interaction potential (1) integral in (74) can be substituted by overall RDFs Laplace transform of chain fluid, hence above formula can be written as follows

$$
\beta f_{H T A}=-\frac{2 \beta \epsilon_{0} r_{0} e^{z^{(0)} R}}{\pi} \sum_{a} \pi \rho_{a} m_{a} \sum_{b} \pi \rho_{b} m_{b} e^{-z^{(0)} R} G_{\text {overall }}^{a b}\left(z^{(0)}\right),
$$

where

$$
G_{\text {overall }}^{a b}\left(z^{(0)}\right)=\int_{0}^{\infty} d r r e^{-z^{(0)} r} g_{\text {overall }}^{a b}(r) .
$$

We will use obtained expression for the Laplace transform of the overall RDF (61) for HTA free energy contribution (75), which now reads

$$
\begin{aligned}
\beta f_{H T A}= & -\frac{2 \beta \epsilon_{0} r_{0}}{\pi \Delta D}\left\{l^{2}\left[\frac{1}{z^{(0)^{2}}}+\frac{R}{z^{(0)}}-\frac{R^{3}}{\Delta z^{(0)}} \frac{\varphi_{1}(R)}{\varphi_{0}(R)} v+\frac{l R^{4}}{4 \Delta z^{(0)}}+\frac{R^{3}}{2 \Delta z^{(0)}}(l+2 v)\right]\right. \\
& +4 l v\left[\frac{1}{z^{(0)^{2}}}+\frac{R}{z^{(0)}}-\frac{R}{\varphi_{0}(R)}\left(\frac{1}{z^{(0)}}+\frac{R}{2 z^{(0)}}\right)-\frac{R^{3}}{\Delta z^{(0)}} \frac{\varphi_{1}(R)}{\varphi_{0}(R)} v+\frac{l R^{4}}{4 \Delta z^{(0)}}+\frac{(l+2 v) R^{3}}{2 \Delta z^{(0)}} \frac{\varphi_{1}(R)}{\varphi_{0}(R)}\right] \\
& +4 v^{2}\left[\left(\frac{\varphi_{1}(R)}{\varphi_{0}(R)}\right)^{2}+R \frac{\varphi_{1}(R)}{\varphi_{0}(R)}+\frac{l R^{4}}{4 \Delta z^{(0)}}+\frac{l+2 v}{\Delta}\left(\left(\frac{R^{3}}{2}+\frac{\varphi_{1}(R) z^{(0)} R^{2}}{2}\right)\left(\frac{\varphi_{1}(R)}{\varphi_{0}(R)}\right)^{2}-R^{2} \varphi_{1}(R) \frac{\varphi_{1}(R)}{\varphi_{0}(R)}\right)\right. \\
& \left.\left.+\frac{\left(l+2 v \frac{\varphi_{1}(R)}{\varphi_{0}(R)} z^{(0)}\right) R^{2} \varphi_{1}(R)}{2 \Delta z^{(0)}}\right]\right\}-\frac{2 \beta \epsilon_{0} r_{0}}{\pi} \frac{2 v}{\varphi_{0}(R)}
\end{aligned}
$$

where

$$
\begin{gathered}
D=1-\frac{2}{\Delta} \varphi_{2}(R)\left(l+2 v \frac{\varphi_{1}(R)}{\varphi_{0}(R)} s\right)\left(1+\frac{R^{3}}{2 \Delta}(l+2 v)\right)+\frac{R^{3} \varphi_{2}(R)}{\Delta^{2}}(l+2 v) 2 v \frac{\varphi_{1}(R)}{\varphi_{0}(R)} s \\
-\frac{1}{\Delta} \varphi_{1}(R) R\left[(l+2 v)\left(1+\frac{l R^{3}}{2 \Delta}\right)+\left(l+2 v \frac{\varphi_{1}(R)}{\varphi_{0}(R)} s\right)\right] \\
l=\pi \sum_{c} \rho^{c} m_{c}, \\
v=\pi \sum_{c} \rho^{c} m_{c} \frac{K_{c}}{1-K_{c}},
\end{gathered}
$$

are the moments of the chain species distribution function, and

$$
\begin{aligned}
& \varphi_{2}\left(R^{(b)}\right)=\frac{1-z^{(0)} R^{(b)}+z^{(0)^{2}} R^{(b)^{2}} / 2-e^{-z^{(0)} R^{(b)}}}{z^{(0)^{3}}} \\
& \varphi_{1}\left(R^{(b)}\right)=\frac{1-z^{(0)} R^{(b)}-e^{-z^{(0)} R^{(b)}}}{z^{(0)^{2}}} \\
& \varphi_{0}\left(R^{(b)}\right)=\frac{1-e^{-z^{(0)} R^{(b)}}}{z^{(0)}} .
\end{aligned}
$$

To study the phase behavior, we will also need expressions for the pressure of the system and chemical potential of the chain molecules, which are derived from the above expression for Helmholtz free energy (69)-(78)

$$
\begin{aligned}
& \beta P=\beta \sum_{c} \rho_{c} \mu_{c}-\beta f=\beta P_{H S}+\beta P_{c h a i n}+\beta P_{H T A}, \\
& \beta \mu_{c}=\frac{\partial(\beta f)}{\partial \rho_{c}}=\beta \mu_{c, i d}+\beta \mu_{c, H S}^{e x}+\beta \mu_{c, \text { chain }}+\beta \mu_{c, H T A} .
\end{aligned}
$$


Here $\mu_{c, i d}, \mu_{c, H S}^{e x}, \mu_{c, c h a i n}, \mu_{c, H T A}$ and $P_{c h a i n}, P_{H T A}$ hold contributions from corresponding free energies $f_{i d}, f_{H S}^{e x}$, $f_{\text {chain }}$ and $f_{H T A}$, except for $\beta P_{H S}$ that holds sum of contributions from $f_{i d}$ and $f_{H S}^{e x}$. These expressions for excess chemical potential and pressure can be formulated in terms of moments and their density derivatives

$$
\begin{aligned}
\beta \mu_{c, i d}= & m_{c} \ln \left\{\Lambda_{c}^{3} \rho_{c}\right\} \\
\beta \mu_{c, H S}^{e x}= & \frac{l R^{3} l(c)}{\pi \Delta}\left(1+\frac{1}{3 \Delta}\right)+\frac{l^{2} R^{6} l(c)}{12 \pi \Delta^{2}}\left(1+\frac{2}{3 \Delta}\right) \\
\beta \mu_{c, \text { chain }}= & -\left(m_{c}-1\right) \ln \left\{4 \pi \rho_{c} R^{2} Y\right\}-\frac{(l-\pi \rho)}{Y} \frac{\partial Y}{\partial \rho_{c}} \\
\beta \mu_{c, H T A}= & -\frac{4 \beta \epsilon_{0} r_{0} e^{z^{(0)} R}}{\pi} \pi \rho_{c} m_{c} \sum_{a} \pi \rho_{a} m_{a} G_{\text {overall }}^{\text {ac }}\left(z^{(0)}\right) \\
\beta P_{H S}= & \frac{l}{\Delta \pi}\left(1+\frac{l R^{3}}{2 \Delta}+\frac{l^{2} R^{6}}{12 \Delta^{2}}+\frac{l^{3} R^{9}}{216 \Delta^{2}}\right) \\
\beta P_{\text {chain }}= & \frac{l-\pi \rho}{\pi}\left(\frac{1}{Y}\left[\frac{5 l \epsilon^{3}}{12 \Delta^{2}}+\frac{l^{2} R^{6}}{12 \Delta^{3}}\right]+1\right) \\
\beta P_{H T A}= & -\frac{2 \beta \epsilon_{0} r_{0} e^{z^{(0)} R}}{\pi} \sum_{a} \sum_{a} \pi \rho_{a} m_{a} \sum_{a} \sum_{b} \pi \rho_{b} m_{b} \frac{\partial \sigma_{b} m_{b} G_{\text {overall }}^{a c}\left(z^{(0)}\right)}{\partial \rho_{c}} \\
& -\frac{2 \beta \epsilon_{0} r_{0} e^{z^{(0)}} R}{\pi} \sum_{a} \pi \rho_{a} m_{a} \sum_{b} \pi \rho_{b} m_{b} \sum_{c} \rho_{c} \frac{\partial G_{\text {overall }}^{a b}\left(z^{(0)}\right)}{\partial \rho_{c}}
\end{aligned}
$$

where

$$
\begin{aligned}
\frac{\partial Y}{\partial \rho_{c}} & =\frac{5 R^{3} l_{c}}{12 \Delta^{3}}+\frac{l R^{6} l_{c}}{9 \Delta^{2}}+\frac{l^{3} R^{9} l_{c}}{144 \Delta^{4}} \\
l_{c} & =\frac{\partial l}{\partial \rho_{c}}=\pi m_{c} \\
v_{c} & =\frac{\partial v}{\partial \rho_{c}}=\pi m_{c} \frac{K_{c}}{1-K_{c}} .
\end{aligned}
$$

and $\rho=\sum_{c} \rho_{c}$ is total number density. Because of the size expressions for $\mu_{c, H T A}$ and $P_{H T A}$ via the moments are shown in the Appendix.

Thus, we can conclude, that our model belongs to the family of the TFE models, since its thermodynamic properties can be represented by finite number of (generalized) density distribution moments.

\section{TWO PHASE EQUILIBRIA}

When at constant $T$ mother phase with the number density $\rho_{c}^{(0)}$ of the chain species $c$ and volume $V^{(0)}$ separates into equilibrium daughter phases, then all the chemical potentials of every chain species and all pressures in each of the daughter phases are equal. Thus for the two-phase equilibrium we have

$$
\begin{aligned}
\mu_{c}^{(1)}\left(\left\{\rho_{c}^{(1)}\right\}\right) & =\mu_{c}^{(2)}\left(\left\{\rho_{c}^{(2)}\right\}\right), \text { for every } c \\
P^{(1)} & =P^{(2)},
\end{aligned}
$$

where $P^{(p)}$ and $\mu_{c}^{(p)}\left(\left\{\rho_{c}^{(p)}\right\}\right)$ are pressure and chemical potential of phase $p$. We have explicitly specified the dependence on the species number densities $\left\{\rho_{c}^{(p)}\right\}$ to indicate that they differ between phases. In addition particle number and volume conservation rules have to be satisfied

$$
\begin{aligned}
& f_{c}^{(0)}=x^{(1)} f_{c}^{(1)}+x^{(2)} f_{c}^{(2)} \\
& v^{(0)}=x^{(1)} v^{(1)}+x^{(2)} v^{(2)},
\end{aligned}
$$


together with normalization condition

$$
1=x^{(1)}+x^{(2)}
$$

where $x^{(p)}=N^{(p)} / N^{(0)}$ for $p=1,2$ is the chain molecules number fractions of phase $p, N^{(p)}=V^{(p)} \rho^{(p)}$ is the total chain molecule number in phase $p$, and $f_{c}^{(p)}=\rho_{c}^{(p)} / \rho^{(p)}=N_{c}^{(p)} / N^{(p)}$ is molecular species distribution function

$$
\sum_{c} f_{c}^{(p)}=1
$$

Assuming that we have the number of the chain species $M$, conditions (96)-(100) form closed set of $M+4$ equations for $M+4$ unknowns: $f_{c}^{(1)}, \rho^{(1)}, \rho^{(2)}, x^{(1)}, x^{(2)}$. As in our previous work $[\underline{6}$, to solve above equations, we will employ general scheme developed by Bellier-Castella et al 23]. The scheme is suitable only for truncated free energy models where very efficient transition to new unknown variables, represented by the moments, can be done. According to the scheme, the set of $M+4$ equations, applied to our model, transforms to 4 equations in two unknown moments of the first phase $l^{(1)}, v^{(1)}$ and two unknown densities of the daughter phases $\rho^{(1)}, \rho^{(2)}$ :

$$
\begin{aligned}
& \rho^{(1)}=\rho^{(1)} \sum_{c} f_{c}^{(0)} H_{c}\left(T ; \rho^{(1)}, l^{(1)} v^{(1)} ; \rho^{(2)}, l^{(2)} v^{(2)}\right), \\
& l^{(1)}=\rho^{(1)} \sum_{c} l_{c} f_{c}^{(0)} H_{c}\left(T ; \rho^{(1)}, l^{(1)} v^{(1)} ; \rho^{(2)}, l^{(2)} v^{(2)}\right), \\
& v^{(1)}=\rho^{(1)} \sum_{c} v_{c} f_{c}^{(0)} H_{c}\left(T ; \rho^{(1)}, l^{(1)} v^{(1)} ; \rho^{(2)}, l^{(2)} v^{(2)}\right), \\
& P^{(1)}\left(T ; \rho^{(1)}, l^{(1)} v^{(1)}\right)=P^{(2)}\left(T ; \rho^{(2)}, l^{(2)} v^{(2)}\right),
\end{aligned}
$$

where

$$
\begin{aligned}
H_{c}\left(T ; \rho^{(1)}, l^{(1)} v^{(1)} ; \rho^{(2)}, l^{(2)} v^{(2)}\right) & =\frac{\left(\rho^{(1)}-\rho^{(2)}\right) A_{c}^{12}\left(T ; \rho^{(1)}, l^{(1)} v^{(1)} ; \rho^{(2)}, l^{(2)} v^{(2)}\right)}{\left(\frac{\rho^{(2)} \rho^{(1)}}{\rho^{(0)}}-\rho^{(2)}\right)+\left(\rho^{(1)}-\frac{\rho^{(2)} \rho^{(1)}}{\rho^{(0)}}\right) A_{c}^{12}\left(T ; \rho^{(1)}, l^{(1)} v^{(1)} ; \rho^{(2)}, l^{(2)} v^{(2)}\right)},(10 \\
A_{c}^{12}\left(T ; \rho^{(1)}, l^{(1)} v^{(1)} ; \rho^{(2)}, l^{(2)} v^{(2)}\right) & =\frac{\rho^{(2)}}{\rho^{(1)}} \exp \left[\mu_{c}^{e x(2)}\left(T ; \rho^{(2)}, l^{(2)} v^{(2)}\right)-\mu_{c}^{e x(1)}\left(T ; \rho^{(1)}, l^{(1)} v^{(1)}\right)\right]
\end{aligned}
$$

and $\mu_{c}^{e x}=\mu_{c}-\mu_{i d, c}$ is excess chemical potential. Note that two moments of the second phase $l^{(2)}$ and $v^{(2)}$ aren't unknown variables, because they can be found from the relation between moments of the phase 0, which are known in advance, and moments of the phase 1

$$
m^{(2)}=\frac{\rho^{(1)}-\rho^{(2)}}{\rho^{(1)}-\rho^{(0)}} m^{(0)}+\frac{\rho^{(2)}-\rho^{(0)}}{\rho^{(1)}-\rho^{(0)}} m^{(1)},
$$

where $m$ is one of the moments $l$ or $v$. Set of equations presented above are used to locate the binodals. To obtain cloud and shadow curves, we consider the limit $\rho^{(2)} \rightarrow \rho^{(0)}$, which employs that second (cloud) phase is very close to parent phase and thus there is only an infinitesimal amount of the first (shadow) phase . In such case it can be easily shown that

$$
\lim _{\rho^{(2)} \rightarrow \rho^{(0)}} H_{c}\left(T ; \rho^{(1)}, l^{(1)} v^{(1)} ; \rho^{(2)}, l^{(2)} v^{(2)}\right)=A_{c}^{12}\left(T ; \rho^{(1)}, l^{(1)} v^{(1)} ; \rho^{(2)}, l^{(2)} v^{(2)}\right)
$$

and therefore in equations (101)-(103), $H_{c}$ should be replaced by $A_{c}^{12}$.

\section{RESULTS AND DISCUSSION}

We will present now phase diagrams for the two systems that differ from each other due to the mother distribution function $f_{c}^{(0)}$. In contrast to the other studies of polydisperse fluids, we assume that distribution function depends on discrete species index $c$, rather than continuous one. But the species number should be large enough to expose properties of polydisperse systems. Each chain species $c$ has length $m_{c}=c$ monomers, for $c=1$...M. In most cases 


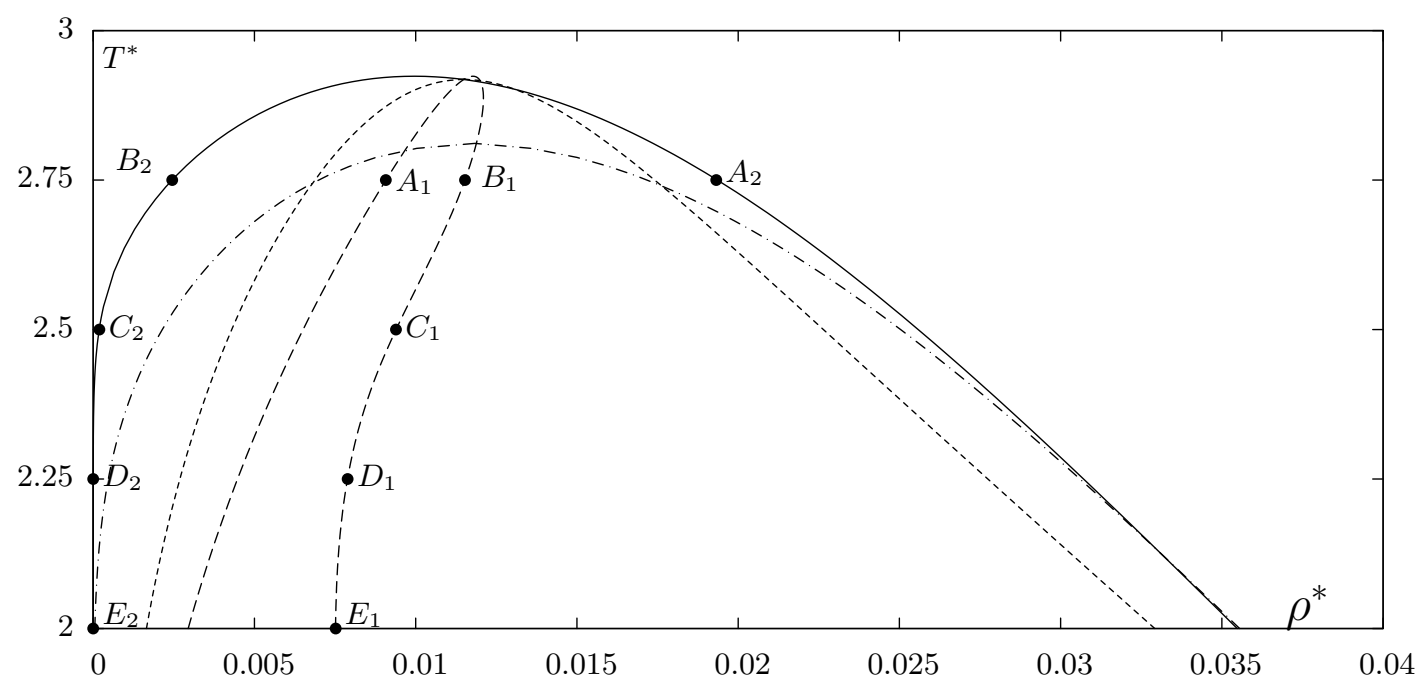

FIG. 1: Phase diagram ( $T^{*}$ vs $\rho^{*}$ ) of polydisperse chain fluid with $D_{m}=0.5$ beta distribution function. Cloud curve, shadow curve are represented by solid and dashed curves respectively. Dotted curve denotes critical binodal, and dash-dotted line represents monodisperse binodal. Also five points on shadow curve $A_{1}, B_{1}, C_{1}, D_{1}, E_{1}$ together with corresponding equilibrium points $A_{2}, B_{2}, C_{2}, D_{2}, E_{2}$ on cloud curve, mark points for which distribution functions are presented on 9

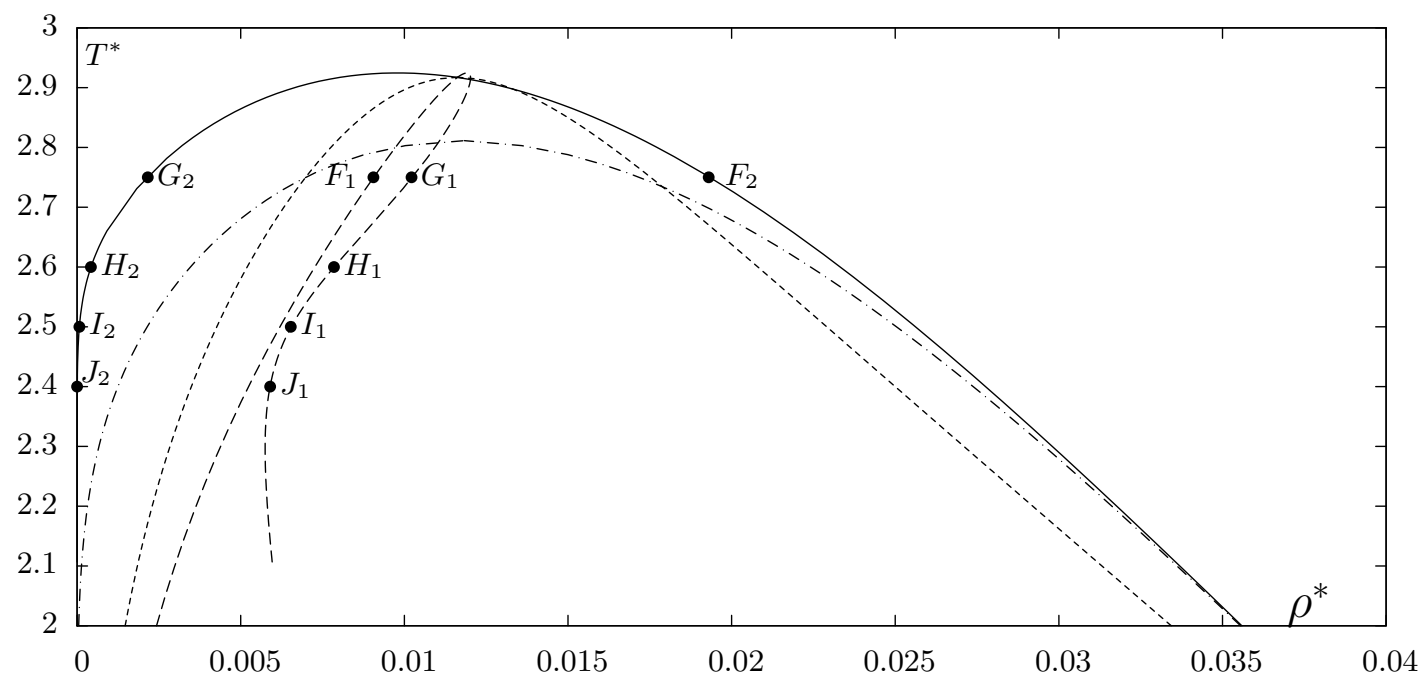

FIG. 2: The same as figure 1 but for Shultz distribution with $z=1$. Corresponding distributions for specified points are presented on [10]

we consider the systems with $M=100$. For the analysis of the cutoff effects we present results for 200 and 300 chain species.

In the following we will consider two systems with two different distribution functions. The first mother distribution function was chosen to be Beta-distribution

$$
f_{c}^{(0)}=\frac{1}{B(\alpha, \beta)}\left(\frac{c}{M}\right)^{\alpha-1}\left(1-\frac{c}{M}\right)^{\beta-1},
$$

for $c=1 . . M$, where $B(\alpha, \beta)$ is beta function and $\alpha$ and $\beta$ are related to mean chain length $\left(m_{0}=\langle m\rangle\right)$ and mean 


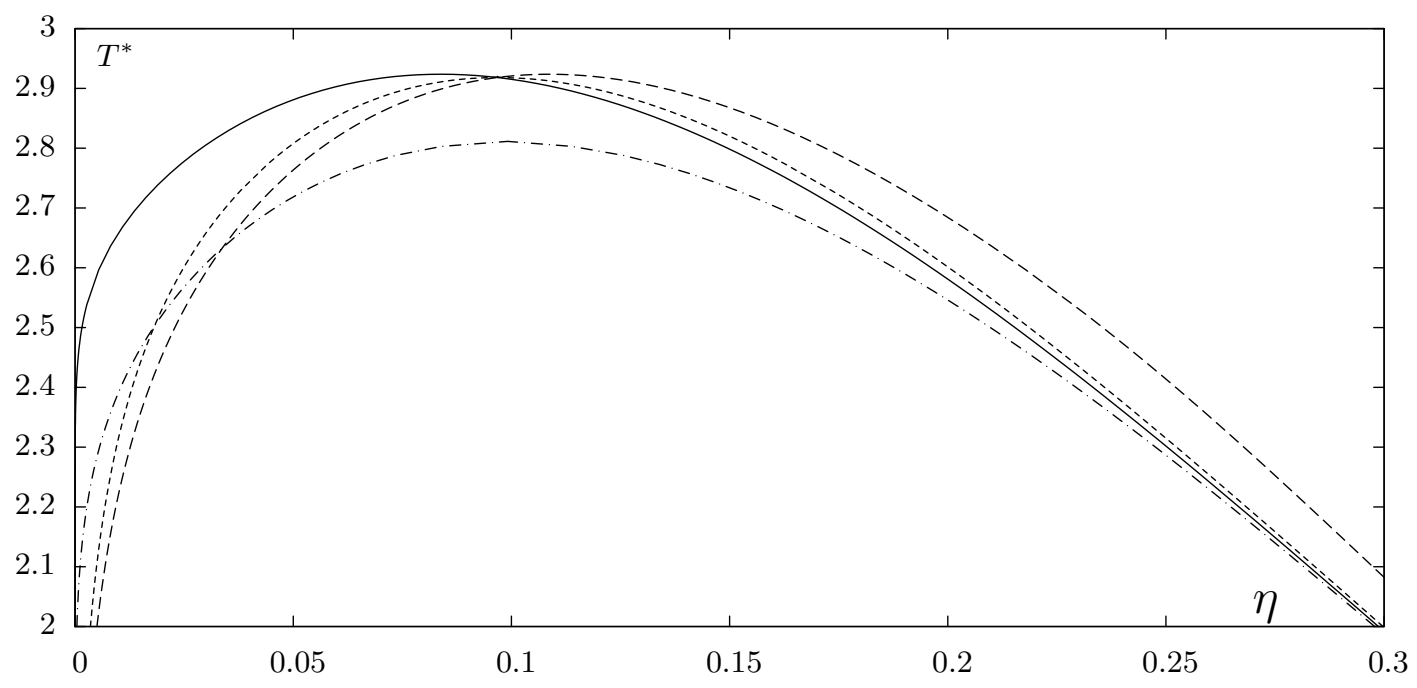

FIG. 3: Phase diagram ( $T^{*}$ vs $\left.\eta=(\pi / 6) R^{3} \sum_{c} m_{c} \rho_{c}\right)$ of polydisperse chain fluid with $D_{m}=0.5$ beta distribution function. The curves denote the same as in 1 and 2

square length $\left\langle m^{2}\right\rangle$ by

$$
\begin{aligned}
& \alpha=\frac{M-m_{0}\left(1+D_{m}\right)}{M D_{m}}, \\
& \beta=\left(\frac{M-m_{0}}{m_{0}}\right) \alpha, \\
& D_{m}=\frac{\left\langle m^{2}\right\rangle}{m_{0}^{2}}-1=\frac{\sigma_{m}^{2}}{m_{0}^{2}},
\end{aligned}
$$

with $\sigma_{m}=\sqrt{\left\langle(m-\langle m\rangle)^{2}\right\rangle}$ being standard chain length deviation.

The second distribution function was chosen to be represented by the Shultz distribution

$$
f_{c}^{(0)}=\frac{1}{z !}\left(\frac{z+1}{m_{0}}\right)^{z+1} c^{z} \exp \left[-\left(\frac{z+1}{m_{0}}\right) c\right]
$$

for $c=1 . . M$, where $z$ is dispersity controlling parameter.

In both cases the dispersity of the distribution functions were chosen to be equal, with standard deviation $\sigma_{m}=11.31$ that corresponds to $D_{m}=0.5$ for beta distribution and $z=1$ for Shultz distribution, and with average chain length $m_{0}=16$ for both distributions.

The corresponding phase diagrams for both systems are demonstrated on figures 1 and 2 in terms of the number densities and on figures 3 and 4 in terms the volume fraction $\eta$. Phase diagrams on figures 3 and 4 are almost indistinguishable, but figures 12 have one significant difference: liquid part of the shadow curve for the system with Shultz distribution has smaller density than that of them phase diagram with Beta distribution. This difference of the phase behavior can be explained by inspecting distribution functions on figures 9 and 10 for corresponding points marked on the phase diagrams on figures 1 and 2 Liquid shadow curves for both systems consist of the chains longer than average, in contrast to the gas shadow curves, that are mainly composed of shorter chains. As one can see for the system with Shultz distribution the chains in liquid phase are on average larger than for the systems with Beta distribution. The origin of these effects lies in the difference of the tails for the mother phase distributions (either Beta or Shultz). For Shultz distribution the tail is "fatter" than for Beta distribution. Being more precise at cutoff distance $m_{\text {cutoff }}=M=100$ the Shultz distribution tends to finite value as opposed to Beta whose value tends to 0 . These differences in compositions of the two liquid shadow phases are the reason for the discrepancy between phase diagrams. Note, that volume fractions are close, only average chain lengths and as a result number densities differ.

Figures $[5$ and 6 show shadow curves and critical binodals in terms of the average chain length $\langle m\rangle$. Average chain length of the cloud curve is always 16. In both cases liquid part of the shadow curve moves to longer chains with the temperature decrease, but for Shultz distributed system this move is more rapid. 


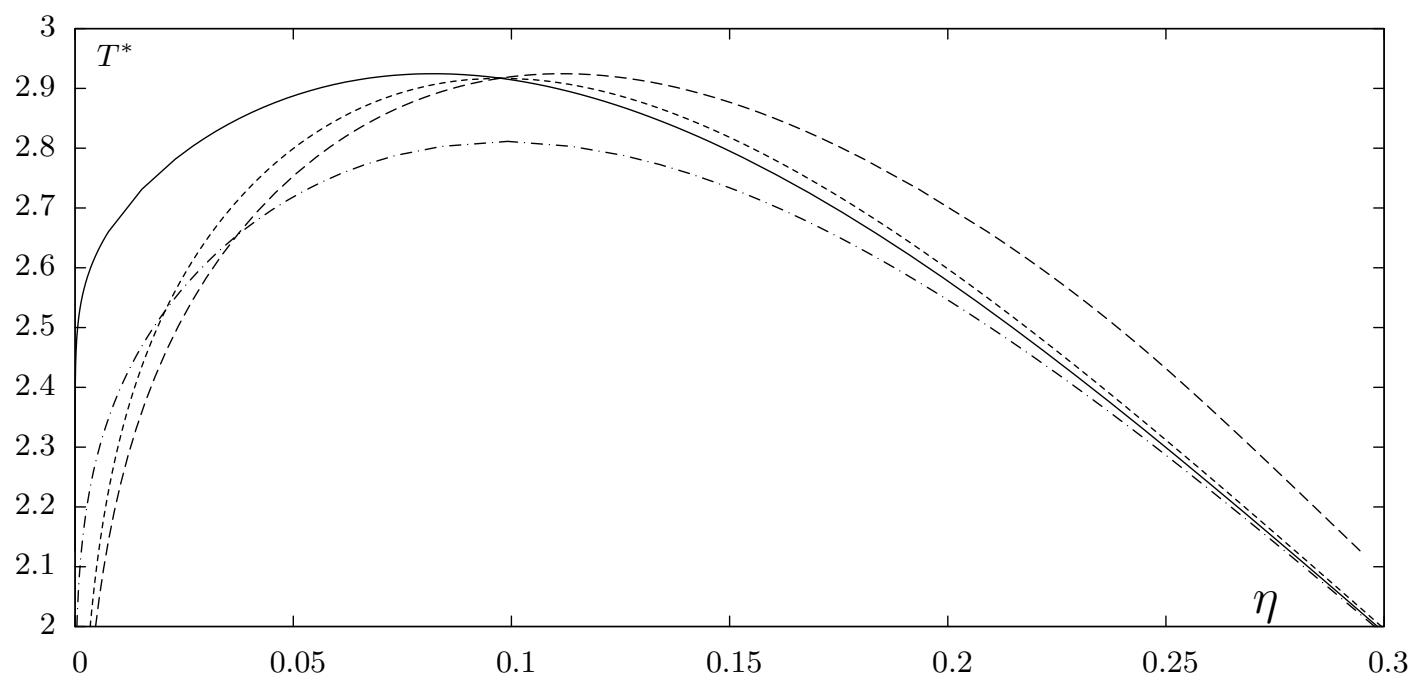

FIG. 4: Same as 3 but for Shultz distribution with $z=1$.

Figures 7 and 8 expose shadow curves and critical binodals in terms of the chain length standard deviation $\sigma_{m}$. Similarly to the average lengths liquid part of the shadow curve is on the right and as the temperature decreases it moves to larger chain length deviations. However after reaching some maximum it starts to decrease. This decrease corresponds to the formation and growing of the peak on the distribution function in the region of long chains. Beta distributed system shows noticeable peak for the point $E_{1}$ on the phase diagram, its distribution function can be found on figure 9 With the temperature decrease the peak grows and approaches the right margin $M=100$ of chain lengths. The peak for the shadow phase of Shultz distributed system has irregular form, its maximum is always at $c=M=100$ (see figure 10 for point $J_{1}$ ) and it also grows with the temperature decrease. These effects indicate that for the low temperatures liquid shadow phase becoming more and more monodisperse and is composed mainly of long chains. The same happends for the gas shadow phase, but it "likes" more short chains than long.

To study conditions at which one can observe irregular peak formation, we examined six additional phase diagrams of Shultz distributed system with $z=5, z=10$ and at three values of the cutoff chain lengths, i.e. the number of species $m_{\text {cutof } f}=M=100,200,300$. They can be found at figure 11 . We can deduce that increase of the cutoff chain length lowers temperature of the peak formation. However this lowering is rather negligible when compared to the change of the peak appearance temperature due to the change of the mother distribution polydispersity. Thus, to get the peak on the distribution function of the liquid shadow phase at high enough temperature one should study the system with high enough polydispersity.

We also examined the change of the critical point position due to the change of the mother phase distribution function polydispersity (figures 12 and 13). For both systems critical point moves to the higher temperatures and to the lower volume fractions with the increase of the distribution function width.

In our previous work [6], which is dedicated to the Yukawa hard spheres with size polydispersity we concluded that hard sphere repulsive interactions plays a major role in controlling fractionation effects. In the present case the situation is different: strong intramolecular attraction may change effective radius of the chain molecules, which are flexible and this may have influence on the phase behavior. But our theory takes structural properties of the system only on the level of hard sphere chains without attraction, and hence it can't capture the effects of effective radius change.

To verify the accuracy of our theory we need to perform molecular MC simulations similar to those made by Wilding at al $[24,25]$.

\section{CONCLUDING REMARKS}

We have shown that polydisperse Yukawa chain mixture with chain length polidispersity treated within HTA belongs to the TFE class of the models, i.e. its thermodynamical properties were shown to depend on the finite set of generalized distribution function moments. Exploiting this property we represent coexistence relations as a set of four nonlinear equations for unknown moments of daughter distribution functions. To illustrate how tails of 


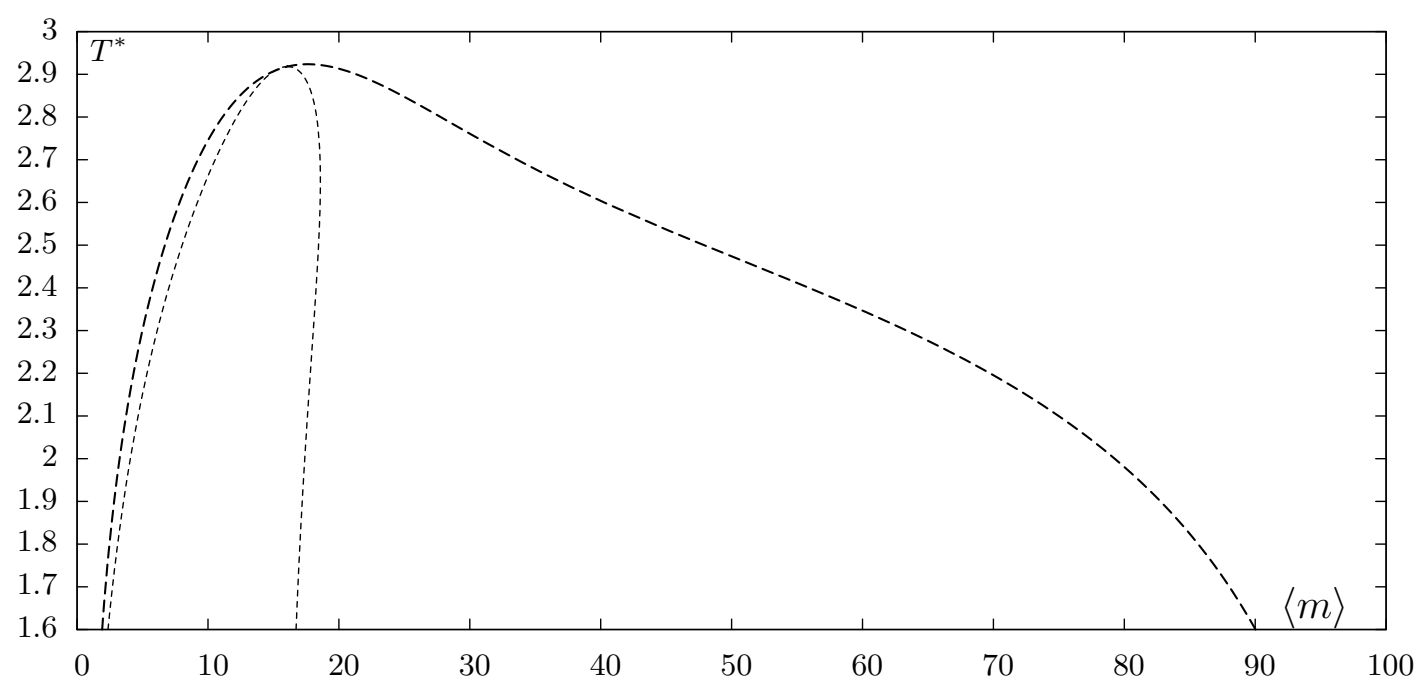

FIG. 5: Shows shadow and critical binodal curves for system with Beta distribution, denoted respectively by dashed and dotted lines, in terms if $T^{*}$ and phase average chain length $\langle m\rangle$.

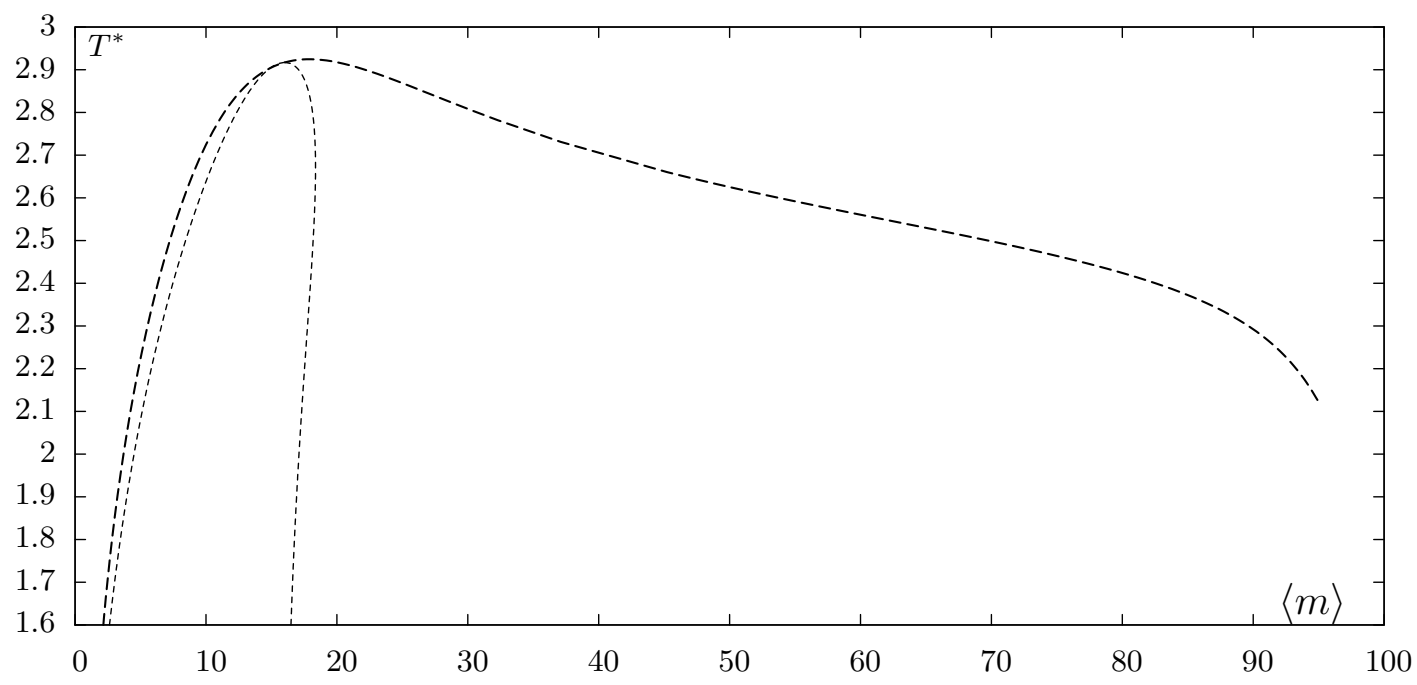

FIG. 6: Same as figure 5ut for Shultz distribution.

the distribution functions can impact the phase behavior these equations were solved and the phase diagrams of the systems with two different mother phase distribution functions with the same dispersion characteristics (mean chain length and standard deviation) and different asymptotics of the distribution function tails were studied. The obtained results show strong dependence of the shadow curve position on the asymptotic of the mother phase distribution function.

We also demonstrated that temperature of the appearance of the peak on the distribution function of the shadow phase strongly depends on the system polydispersity.

\section{APPENDIX A: EXPRESSIONS FOR THE CHEMICAL POTENTIAL AND PRESSURE}

The quantities given below can be simplified, but they are kept her in the form that resembles the structure of the overall RDFs Laplace transform. 


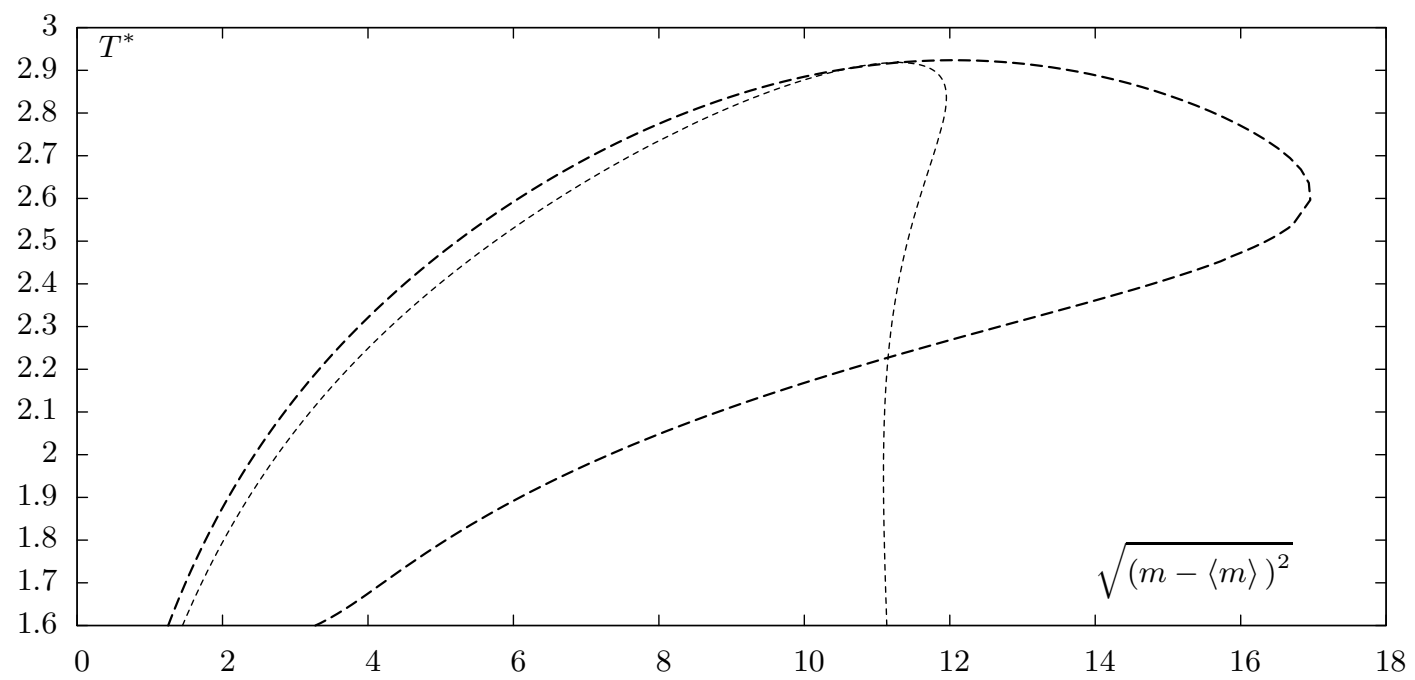

FIG. 7: The same as [5 but reexpressed in terms of chain length standard deviation $\sigma_{m}=\sqrt{(m-\langle m\rangle)^{2}}$.

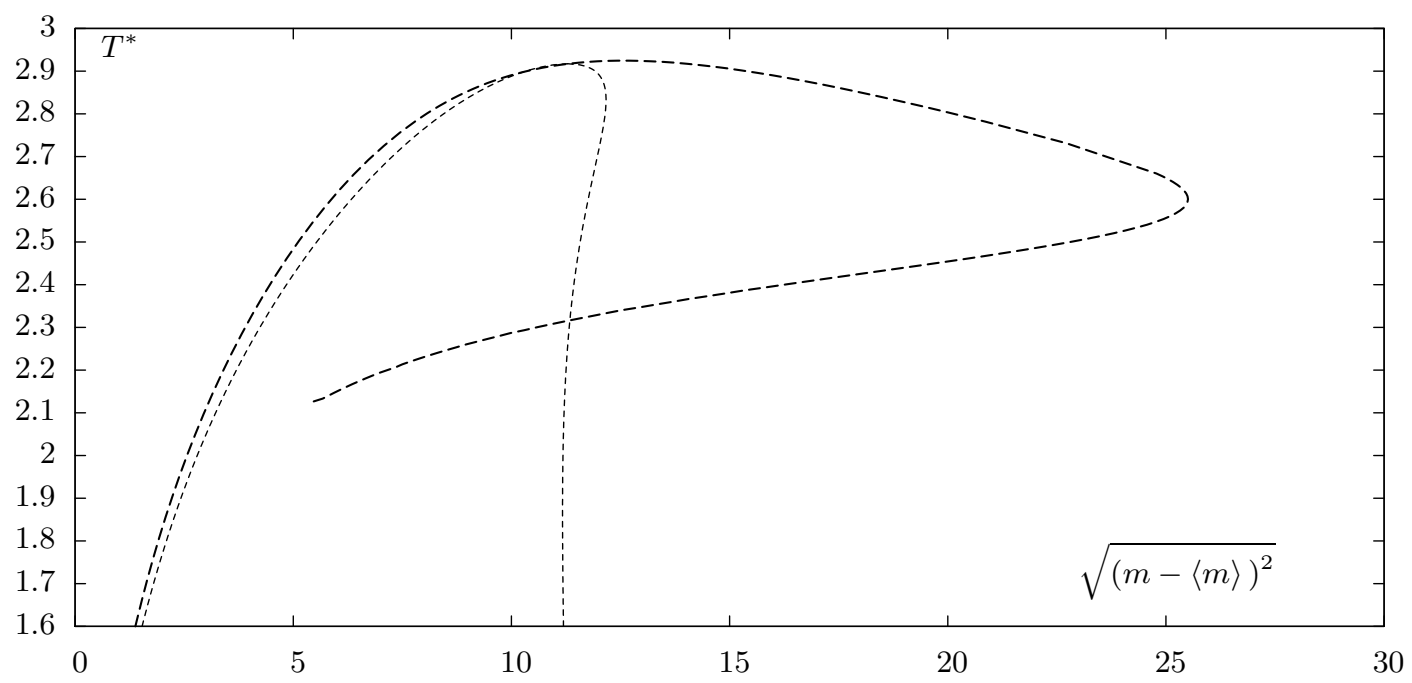

FIG. 8: Same as figure[7 but for Shultz distribution. 


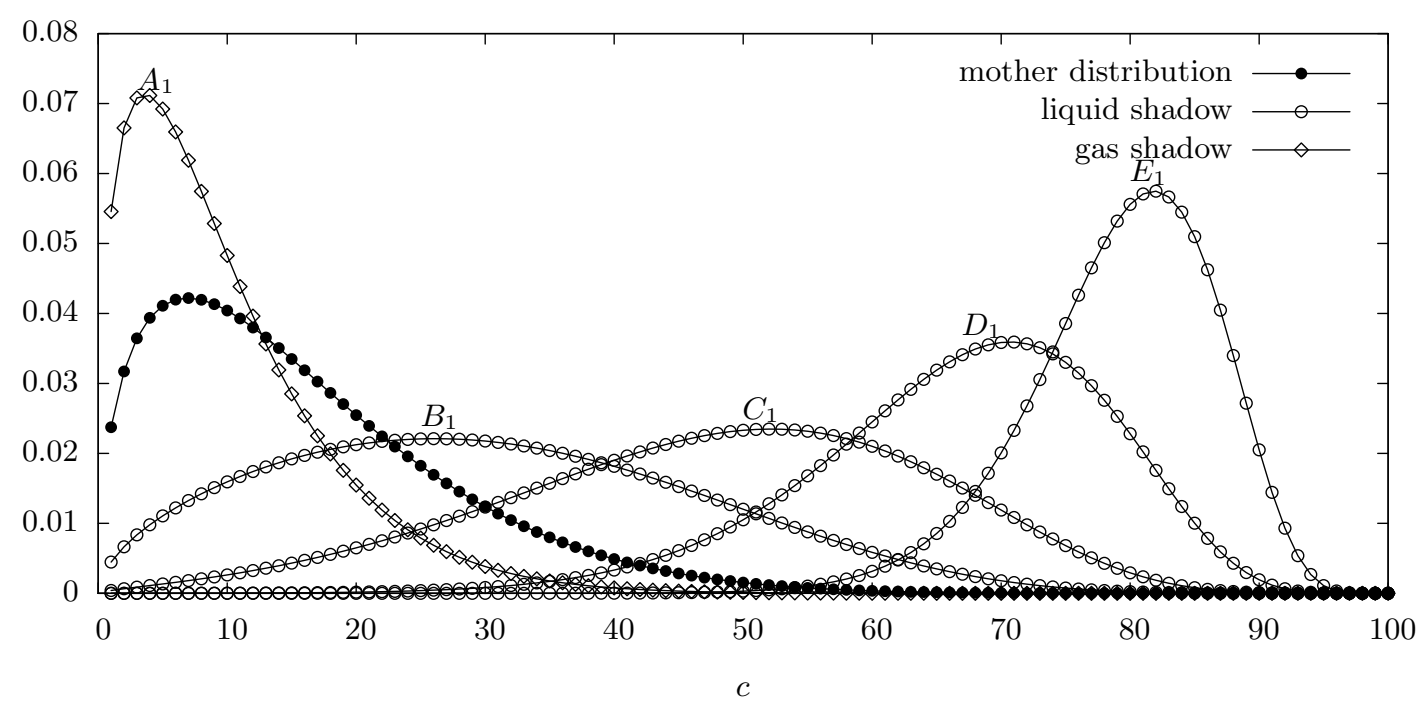

FIG. 9: Distribution functions $f_{c}=\rho_{c} / \rho$ of Beta distributed system of five points, marked on 1 along shadow curve, together with cloud curve (mother) distribution.

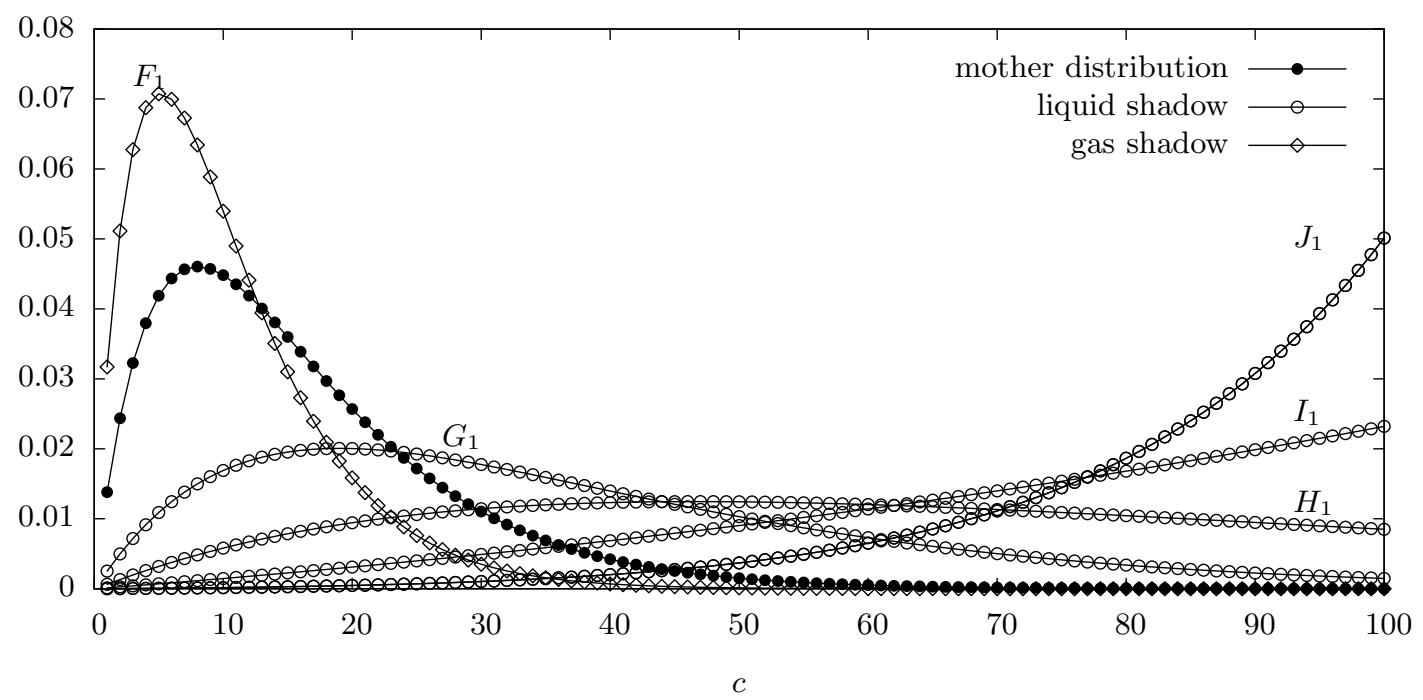

FIG. 10: Same as figure 9 but for system with Shultz distribution, and phase diagram, containing the points, given on figure 2 


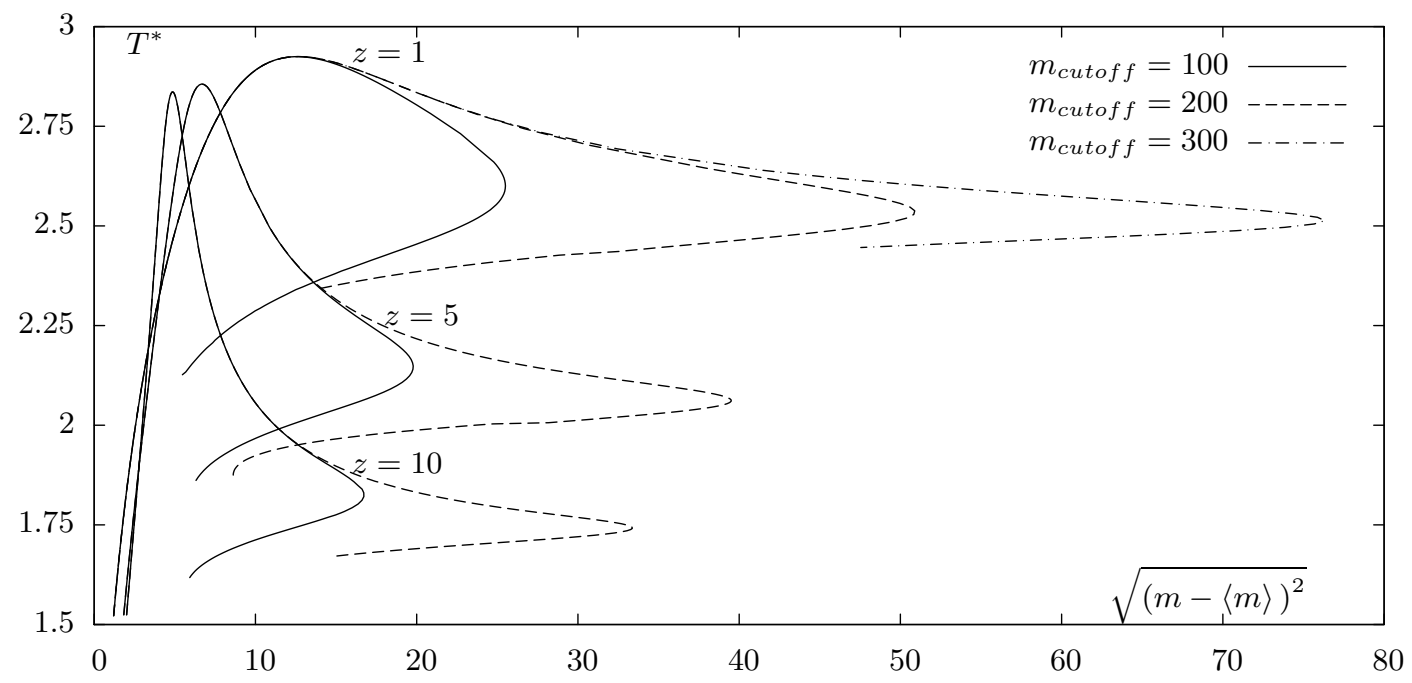

FIG. 11: Figure shows shadow curves of system with Shultz distribution for $z=1, z=5$ and $z=10$ and different cutoffs of distribution function, i.e. different maximal species chain lengths 100,200 and 300. The figure is displayed in terms of $T^{*}$ and standard deviation of chain length $\sigma_{m}=\sqrt{(m-\langle m\rangle)^{2}}$. The decrease of chain length deviation with decrease of temperature that is located below chain length deviation maximum, corresponds to formation of distribution function peak near the cutoff distance. The peak appears in liquid branch of shadow curve.

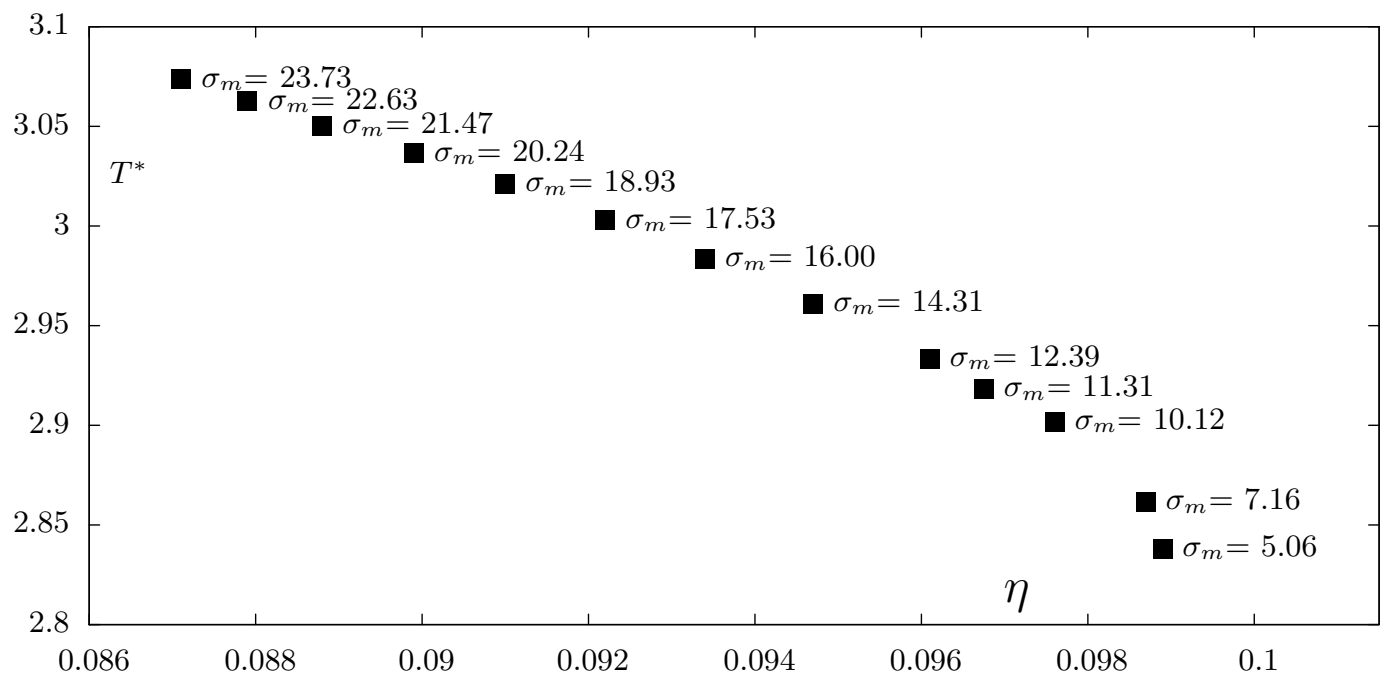

FIG. 12: Critical temperatures and volume fractions $\eta$ for variety of Beta distributions dispersions. 


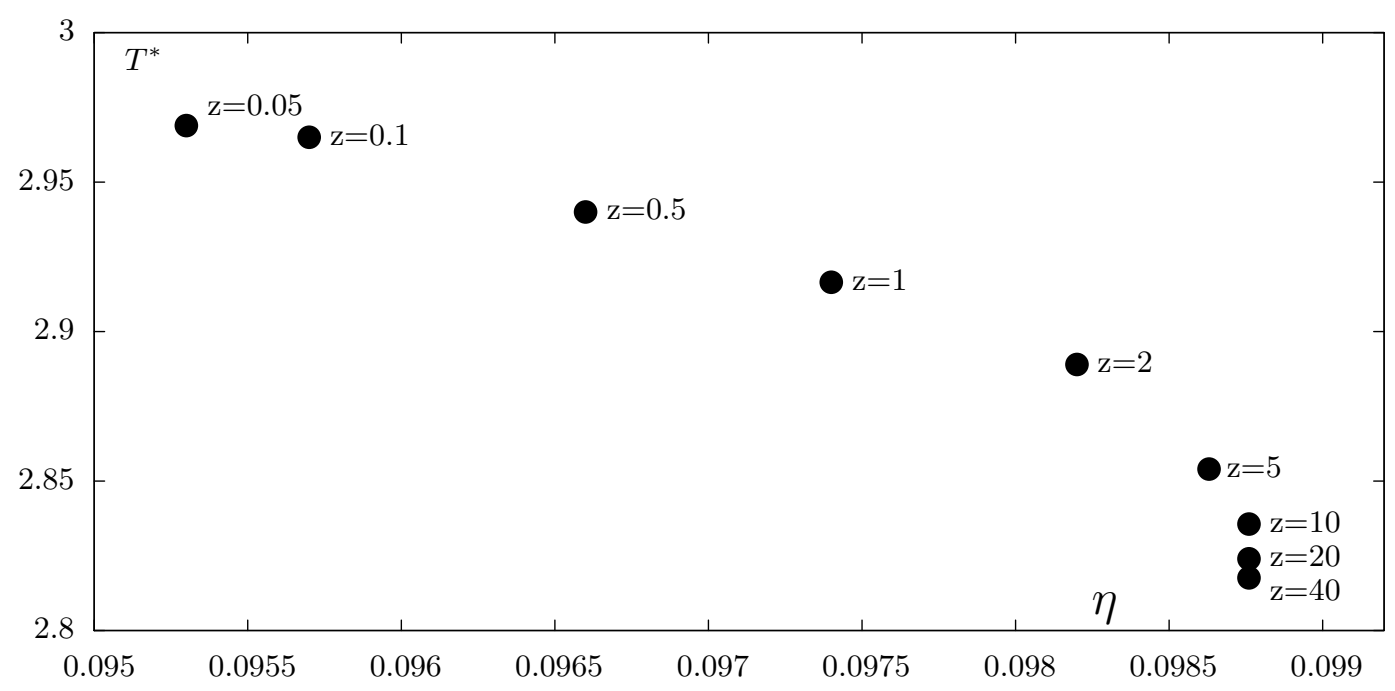

FIG. 13: Critical temperatures and volume fractions $\eta$ for variety of Shultz distributions dispersions.

Contribution from HTA free energy term to chemical potential reads

$$
\begin{aligned}
& \beta \mu_{c, H T A}=-\frac{4 \beta \epsilon_{0} r_{0}}{\pi \Delta D}\left\{l l_{c}\left[\frac{1}{z^{(0)^{2}}}+\frac{R}{z^{(0)}}-\frac{R^{3}}{\Delta z^{(0)}} \frac{\varphi_{1}(R)}{\varphi_{0}(R)} v+\frac{l R^{4}}{4 \Delta z^{(0)}}+\frac{R^{3}}{2 \Delta z^{(0)^{2}}}(l+2 v)\right]\right. \\
& +2\left(v l_{c}+l v_{c}\right)\left[\frac{1}{z^{(0)^{2}}}+\frac{R}{z^{(0)}}-\frac{R}{\varphi_{0}(R)}\left(\frac{1}{z^{(0)^{2}}}+\frac{R}{2 z^{(0)}}\right)-\frac{R^{3}}{\Delta z^{(0)}} \frac{\varphi_{1}(R)}{\varphi_{0}(R)}+\frac{l R^{4}}{4 \Delta z^{(0)}}+\frac{(l+2 v) R^{3}}{2 \Delta z^{(0)}} \frac{\varphi_{1}(R)}{\varphi_{0}(R)}\right] \\
& +4 v v_{c}\left[\left(\frac{\varphi_{1}(R)}{\varphi_{0}(R)}\right)^{2}+R \frac{\varphi_{1}(R)}{\varphi_{0}(R)}+\frac{l R^{4}}{4 \Delta z^{(0)}}+\frac{l+2 v}{\Delta}\left(\left(\frac{R^{3}}{2}+\frac{\varphi_{1}(R) z^{(0)} R^{2}}{2}\right)\left(\frac{\varphi_{1}(R)}{\varphi_{0}(R)}\right)^{2}-R^{2} \varphi_{1}(R) \frac{\varphi_{1}(R)}{\varphi_{0}(R)}\right)\right. \\
& \left.\left.+\frac{\left(l+2 v \frac{\varphi_{1}(R)}{\varphi_{0}(R)} z^{(0)}\right) R^{2} \varphi_{1}(R)}{2 \Delta z^{(0)}}\right]\right\}-\frac{4 \beta \epsilon_{0} r_{0}}{\pi} \frac{v_{c}}{\varphi_{0}(R)} \\
& +\beta\left(f_{H T A}-f_{H T A}^{i n t r a}\right)\left(\frac{l_{c} R^{3}}{6 \Delta}-\frac{\frac{\partial D}{\partial \rho_{c}}}{D}\right) \\
& -\frac{2 \beta \epsilon_{0} r_{0}}{\pi \Delta D}\left\{l l \left[-\frac{v_{c} R^{3}}{\Delta z^{(0)}} \frac{\varphi_{1}(R)}{\varphi_{0}(R)}-\frac{v R^{6} l_{c}}{6 \Delta^{2} z^{(0)}} \frac{\varphi_{1}(R)}{\varphi_{0}(R)}+\frac{l_{c} R^{4}}{4 \Delta z^{(0)}}+\frac{l R^{7} l_{c}}{24 \Delta^{2} z^{(0)}}+\frac{R^{3}\left(l_{c}+2 v_{c}\right)}{2 \Delta z^{(0)}}+\frac{(l+2 v) R^{6} l_{c}}{\left.12 \Delta^{2} z^{(0)^{2}}\right]}\right.\right. \\
& +4 l v\left[-\frac{R^{3} v_{c}}{\Delta z^{(0)}} \frac{\varphi_{1}(R)}{\varphi_{0}(R)}-\frac{v R^{6} l_{c}}{6 \Delta^{2} z^{(0)}} \frac{\varphi_{1}(R)}{\varphi_{0}(R)}+\frac{l_{c} R^{4}}{4 \Delta z^{(0)}}+\frac{l R^{7} l_{c}}{24 \Delta^{2} z^{(0)}}+\left(\frac{R^{3}\left(l_{c}+2 v_{c}\right)}{2 \Delta z^{(0)}}+\frac{(l+2 v) R^{6} l_{c}}{12 \Delta^{2} z^{(0)}}\right) \frac{\varphi_{1}(R)}{\varphi_{0}(R)}\right] \\
& +4 v v\left[\frac{l_{c} R^{4}}{4 \Delta z^{(0)}}+\frac{l R^{7} l_{c}}{24 \Delta^{2} z^{(0)}}+\left(\frac{l_{c}+2 v_{c}}{\Delta}+\frac{(l+2 v) R^{3} l_{c}}{6 \Delta^{2}}\right)\left(\left(\frac{R^{3}}{2}+\frac{\varphi_{1}(R) z^{(0)} R^{2}}{2}\right)\left(\frac{\varphi_{1}(R)}{\varphi_{0}(R)}\right)^{2}-R^{2} \varphi_{1}(R) \frac{\varphi_{1}(R)}{\varphi_{0}(R)}\right)\right. \\
& \left.+\frac{\left(l_{c}+2 v_{c} \frac{\varphi_{1}(R)}{\varphi_{0}(R)} z^{(0)}\right) R^{2} \varphi_{1}(R)}{2 \Delta z^{(0)}}+\frac{\left(l+2 v \frac{\varphi_{1}(R)}{\varphi_{0}(R)} z^{(0)}\right) R^{5} \varphi_{1}(R) l_{c}}{12 \Delta^{2}(0)}\right]+\frac{2 \beta \epsilon_{0} r_{0}}{\pi} \frac{v_{c}}{\varphi_{0}(R)},
\end{aligned}
$$

where

$$
\beta f_{H T A}^{i n t r a .}=-\frac{2 \beta \epsilon_{0} r_{0}}{\pi} \frac{2 v}{\varphi_{0}(R)}
$$


is contribution from intramolecular Yukawa interactions to free energy (78) and $\partial D / \partial \rho_{c}$ has the following form

$$
\begin{aligned}
\frac{\partial D}{\partial \rho_{c}}= & -\frac{\varphi_{2}(R) R^{3} l_{c}}{3 \Delta^{2}}\left(l+2 v \frac{\varphi_{1}(R)}{\varphi_{0}(R)} z^{(0)}\right)\left(1+\frac{R^{3}}{2 \Delta}(l+2 v)\right)-\frac{2}{\Delta} \varphi_{2}(R)\left[\left(l_{c}+2 v_{c} \frac{\varphi_{1}(R)}{\varphi_{0}(R)} s\right)\left(1+\frac{R^{3}}{2 \Delta}(l+2 v)\right)\right. \\
& \left.+\left(l+2 v \frac{\varphi_{1}(R)}{\varphi_{0}(R)} s\right)\left(\frac{R^{6} l_{c}}{12 \Delta^{2}}(l+2 v)+\frac{R^{3}}{2 \Delta}\left(l_{c}+2 v_{c}\right)\right)\right]+\frac{\varphi_{2}(R) R^{6} l_{c}}{3 \Delta^{3}}(l+2 v) 2 v \frac{\varphi_{1}(R)}{\varphi_{0}(R)} z^{(0)} \\
& +\frac{R^{3} \varphi_{2}(R)}{\Delta^{2}}\left[\left(l_{c}+2 v_{c}\right) 2 v+(l+2 v) 2 v_{c}\right] \frac{\varphi_{1}(R)}{\varphi_{0}(R)} z^{(0)}-\frac{R^{3} l_{c}}{3 \Delta^{2}} \varphi_{1}(R) R\left[(l+2 v)\left(1+\frac{l R^{3}}{2 \Delta}\right)+\left(l+2 v \frac{\varphi_{1}(R)}{\varphi_{0}(R)} z^{(0)}\right)\right] \\
& -\frac{1}{\Delta} \varphi_{1}(R) R\left[\left(l_{c}+2 v_{c}\right)\left(1+\frac{l R^{3}}{2 \Delta}\right)+(l+2 v)\left(\frac{R^{3} l_{c}}{2 \Delta}+\frac{l R^{6} l_{c}}{12 \Delta^{2}}\right)+\left(l_{c}+2 v_{c} \frac{\varphi_{1}(R)}{\varphi_{0}(R)} z^{(0)}\right)\right] .
\end{aligned}
$$

Corresponding pressure expression is given by

$$
\begin{aligned}
& \beta P_{H T A}=\beta f_{H T A}+\beta\left(f_{H T A}-f_{H T A}^{\text {intra. }}\right)\left(\frac{l R^{3}}{6 \Delta}-\frac{\sum_{c} \rho_{c} \frac{\partial D}{\partial \rho_{c}}}{D}\right) \\
& -\frac{2 \beta \epsilon_{0} r_{0}}{\pi \Delta D}\left\{l l \left[-\frac{v R^{3}}{\Delta z^{(0)}} \frac{\varphi_{1}(R)}{\varphi_{0}(R)}-\frac{v R^{6} l}{6 \Delta^{2} z^{(0)}} \frac{\varphi_{1}(R)}{\varphi_{0}(R)}+\frac{l R^{4}}{4 \Delta z^{(0)}}+\frac{l R^{7} l}{24 \Delta^{2} z^{(0)}}+\frac{R^{3}(l+2 v)}{\left.2 \Delta z^{(0)^{2}}+\frac{(l+2 v) R^{6} l}{12 \Delta^{2} z^{(0)^{2}}}\right]}\right.\right. \\
& +4 l v\left[-\frac{R^{3} v}{\Delta z^{(0)}} \frac{\varphi_{1}(R)}{\varphi_{0}(R)}-\frac{v R^{6} l}{6 \Delta^{2} z^{(0)}} \frac{\varphi_{1}(R)}{\varphi_{0}(R)}+\frac{l R^{4}}{4 \Delta z^{(0)}}+\frac{l R^{7} l}{24 \Delta^{2} z^{(0)}}+\left(\frac{R^{3}(l+2 v)}{2 \Delta z^{(0)}}+\frac{(l+2 v) R^{6} l}{12 \Delta^{2} z^{(0)}}\right) \frac{\varphi_{1}(R)}{\varphi_{0}(R)}\right] \\
& +4 v v\left[\frac{l R^{4}}{4 \Delta z^{(0)}}+\frac{l R^{7} l}{24 \Delta^{2} z^{(0)}}+\left(\frac{l+2 v}{\Delta}+\frac{(l+2 v) R^{3} l}{6 \Delta^{2}}\right)\left(\left(\frac{R^{3}}{2}+\frac{\varphi_{1}(R) z^{(0)} R^{2}}{2}\right)\left(\frac{\varphi_{1}(R)}{\varphi_{0}(R)}\right)^{2}-R^{2} \varphi_{1}(R) \frac{\varphi_{1}(R)}{\varphi_{0}(R)}\right)\right. \\
& \left.\left.+\frac{\left(l+2 v \frac{\varphi_{1}(R)}{\varphi_{0}(R)} z^{(0)}\right) R^{2} \varphi_{1}(R)}{2 \Delta z^{(0)}}+\frac{\left(l+2 v \frac{\varphi_{1}(R)}{\varphi_{0}(R)} z^{(0)}\right) R^{5} \varphi_{1}(R) l}{12 \Delta^{2} z^{(0)}}\right]\right\}+\frac{2 \beta \epsilon_{0} r_{0}}{\pi} \frac{v}{\varphi_{0}(R)},
\end{aligned}
$$

where $\sum_{c} \rho_{c} \frac{\partial D}{\partial \rho_{c}}$ can be obtained from a by simple replacing of all $l_{c}$ and $v_{c}$ by $l=\sum_{c} \rho_{c} l_{c}$ and $v=\sum_{c} \rho_{c} v_{c}$.

[1] Yu.V.Kalyuzhnyi, and G.Kahl, J.Chem.Phys. 119, 7335(2003).

[2] Yu.V.Kalyuzhnyi, G.Kahl, and P.T.Cummings, J.Chem.Phys. 120, 10133(2004).

[3] Yu.V.Kalyuzhnyi, G.Kahl, and P.T.Cummings, Europhys.Lett., 72, 96(2005).

[4] Yu.V.Kalyuzhnyi, G.Kahl, and P.T.Cummings, J.Chem.Phys. 123, 124501(2005).

[5] Yu.V.Kalyuzhnyi, and P.T.Cummings, J.Chem.Phys. 124, 114509(2006).

[6] Yu.V.Kalyuzhnyi, and S.P.Hlushak, J.Chem.Phys. 125, 034501(2006).

[7] J. A. Barker, and D. Henderson, Rev. Mod. Phys. 48, 587(1976).

[8] J.-P. Hansen, and I. R. McDonald, Theory of Simple Liquids, Acad. Press, London (1990).

[9] Yu.V.Kalyuzhnyi, C.-T.Lin, and G.Stell, J.Chem.Phys. 106, 1940(1997); ibid 108, 6513, 6525(1998),

[10] G.Stell, C.-T.Lin, and Yu.V.Kalyuzhnyi, J.Chem.Phys. 110, 5444(1999).

[11] C.-T.Lin, G.Stell, and Yu.V.Kalyuzhnyi, J.Chem.Phys. 112, 3071(2000).

[12] M.S.Wertheim, J.Chem.Phys. 87, 7323(1987).

[13] P.J.Whitehead, S.J.Mills, A.Gil-Villegas, A.Galindo, and G.Jackson, J.Chem.Phys. 106, 4168(1997).

[15] J.Chang, and S.I.Sandler, J.Chem.Phys. 102, 437(1995).

[15] J.Chang, and S.I.Sandler, J.Chem.Phys. 102, 437(1995).

[16] J.Chang, and S.I.Sandler, J.Chem.Phys. 103, 3196(1995).

[17] R.J.Baxter, J.Chem.Phys. 52, 4559(1970).

[18] L.Blum, and J.S.Hoye, J.Chem.Phys. 81, 1311(1977).

[19] M.F.Golovko, and I.A.Protsykevich, Chem.Phys.Lett. 142, 463(1987).

[20] M.F.Holovko, and I.A.Protsykevich, Cond.Matt.Phys. 10, 137(1997).

[21] E.Whitebay, P.T.Cummings, and Yu.V.Kalyuzhnyi, C.McCabe, J.Chem.Phys. 121, 8128(2004).

[22] K.E.Starling, G.A.Mansoori, N.F.Carnahan, and Jr.T.W.Leland, J.Chem.Phys. 54, 1523(1971).

[23] L.Bellier-Castella, H.Xu, and M.Baus, J.Chem.Phys. 113 8337(2000).

[24] M.Fasolo, N.B.Wilding, and P.Sollich, J.Chem.Phys. 121, 6887(2004).

[25] P.Sollich, N.B.Wilding, M.Buzzacchi, and M.Fasolo, J.Chem.Phys. 125, 014908(2006). 